\title{
ARTICLE
}

Received 24 Mar 2014 | Accepted 1 Aug 2014 | Published 15 Sep $2014 \quad$ DOl: 10.1038/ncomms5898

\section{Classification of stable three-dimensional Dirac semimetals with nontrivial topology}

\author{
Bohm-Jung Yang $^{1} \&$ Naoto Nagaosa $a^{1,2}$
}

A three-dimensional (3D) Dirac semimetal (SM) is the 3D analogue of graphene having linear energy dispersion around Fermi points. Owing to the nontrivial topology of electronic wave functions, the 3D Dirac SM shows nontrivial physical properties and hosts various exotic quantum states such as Weyl SMs and topological insulators under proper external conditions. There are several kinds of Dirac SMs proposed theoretically and partly confirmed experimentally, but its unified picture is still missing. Here we propose a general framework to classify stable 3D Dirac SMs in systems having the time-reversal, inversion and uniaxial rotational symmetries. We show that there are two distinct classes of 3D Dirac SMs. In one class, the Dirac SM possesses a single Dirac point (DP) at a time-reversal invariant momentum on the rotation axis. Whereas the other class of Dirac SMs have a pair of DPs created by band inversion, and carry a quantized topological invariant.

\footnotetext{
${ }^{1}$ RIKEN Center for Emergent Matter Science (CEMS), Wako, Saitama 351-0198, Japan. ${ }^{2}$ Department of Applied Physics, University of Tokyo, Tokyo 113-8656, Japan. Correspondence and requests for materials should be addressed to B.-J.Y. (email: bjyang@riken.jp).
} 
Dirac semimetal (SM) indicates a phase whose lowenergy excitations can be described by pseudorelativistic Dirac fermions with linear energy dispersion. Before the discovery of three-dimensional (3D) topological insulators ${ }^{1,2}$, graphene $^{3}$ has been considered as the unique system where the intriguing properties of two-dimensional (2D) Dirac fermions can be observed. However, the recent progress in the field of topological insulators has shown that stable 2D Dirac fermions exist ubiquitously on the surface of $3 \mathrm{D}$ topological insulators ${ }^{1,2}$. Moreover, through the careful studies on the topological phase transition between a 3D topological insulator and a normal insulator ${ }^{4-8}$, it is demonstrated that even the $3 \mathrm{D}$ Dirac fermions with the linear dispersion in all three momentum directions can be observed in the same material if we can reach the quantum critical point. As the 3D Dirac point (DP) with fourfold degeneracy does not carry a Chern number, the degeneracy at the gap-closing point can be easily lifted by small external perturbations, hence, the $3 \mathrm{D}$ Dirac fermions can be observed only at the single quantum critical point. However, the approach to the quantum critical point requires the intricate finetuning of the alloy chemical compositions ${ }^{7,8}$, which limits the accessibility to the fascinating physics of $3 \mathrm{D}$ Dirac fermions in experiments.

The breakthrough in the search for stable 3D Dirac SMs is achieved in the recent series of studies on $\mathrm{Na}_{3} \mathrm{Bi}($ refs 9,10) and $\mathrm{Cd}_{3} \mathrm{As}_{2}$ (refs 11-14) compounds, where a pair of 3D DPs stably exist on the $k_{z}$ axis. The stability of the 3D DP in these materials stems from the fact that the system has additional crystalline symmetries other than the time-reversal symmetry (TRS) and inversion symmetry (IS) ${ }^{15-20}$. For instance, Young et al. ${ }^{15,16}$ have proposed that particular space groups allow 3D DPs as symmetry protected degeneracies. Also, Wang et al. have shown the symmetry protection of the 3D DPs through the detailed symmetry analysis of $\mathrm{Na}_{3} \mathrm{Bi}$ (ref. 17) and $\mathrm{Cd}_{3} \mathrm{As}_{2}$ (ref. 18).

In the present paper, we propose a general framework to classify stable 3D Dirac SMs in systems with TRS, IS and uniaxial rotation symmetry, which are the most common symmetries of crystalline solids. Through the careful examination of the condition for the accidental band crossing (ABC), we have accomplished the complete classification of stable 3D Dirac SMs, and uncovered that there are two broad classes of 3D Dirac SMs. The Dirac SMs belonging to the first class are created via a band inversion, and have a pair of DPs on the rotation axis away from the time-reversal invariant momentum (TRIM). On the other hand, the second class of Dirac SMs have a single DP at a TRIM on the rotation axis where the band crossing is ensured by the lattice symmetry. In particular, we find that the first class of 3D Dirac SMs have nontrivial topological properties. We have clarified the fundamental relationship between the crystalline symmetries and the topological property of 3D Dirac SMs, and demonstrated that the 3D topological Dirac SM generally mediates the topological quantum phase transition between a normal insulator and a 3D weak topological insulator (or a topological crystalline insulator). In fact, the $3 \mathrm{D}$ topological Dirac SM itself is a parent state of $3 \mathrm{D}$ topological insulators, which turns into either a $3 \mathrm{D}$ strong topological insulator ${ }^{17,18}$ or a $3 \mathrm{D}$ topological crystalline insulator when the 3D DP acquires a mass gap because of symmetry breaking.

\section{Results}

Basic principles to create 3D Dirac semimetals. Our strategy to synthesize a 3D Dirac SM is as follows. Let us consider a system having both the TRS and IS. In general, the TRS requires $E_{n, \uparrow}(\mathbf{k})=E_{n, \downarrow}(-\mathbf{k})$, where $E_{n, \sigma}(\mathbf{k})$ indicates the energy eigenvalue of the $n$-th band with the spin $\sigma=\uparrow, \downarrow$ at the momentum $\mathbf{k}$.
On the other hand, the IS requires $E_{n, \sigma}(\mathbf{k})=E_{n, \sigma}(-\mathbf{k})$. Therefore, under the combined operation of TRS and IS, $E_{n, \uparrow}(\mathbf{k})=E_{n, \downarrow}(\mathbf{k})$, hence, the energy band is doubly degenerate locally at each $\mathbf{k}$. Under this condition, whenever an $\mathrm{ABC}$ occurs between the valence and conduction bands, a 3D DP with fourfold degeneracy can be generated. According to Murakami et al. ${ }^{4-6}$, such an ABC can be achieved only under certain limited conditions because of the strong repulsion between degenerate bands. Namely, only when the valence and conduction bands have the opposite parities, an ABC can occur at a TRIM by tuning an external parameter $m$. In this case, a 3D DP appears at the quantum critical point $\left(m=m_{c}\right)$ between a normal insulator and a $Z_{2}$ topological insulator (see Fig. 1a). As a band gap opens immediately once $m \neq m_{\mathrm{c}}$, the DP is unstable. However, in many crystals, the rotational symmetry as well as TRS and IS presents ubiquitously and constrains the physical properties of materials. Surprisingly, as we will describe in detail below, the additional uniaxial rotational symmetry strongly modifies the condition for ABC, which allows the 3D Dirac SM to emerge as a stable phase. This is possible because when the valence and conduction bands have different rotation eigenvalues, the level repulsion between them can be significantly relaxed as pointed out by Wang et al. ${ }^{17,18}$, which eventually leads to the emergence of a stable 3D Dirac SM phase in the wide range of the parameter space (see Fig. 1b, c).

To describe an ABC of two bands, each of which is doubly degenerate because of the simultaneous presence of TRS and IS, a $4 \times 4$ matrix Hamiltonian can be used as a minimal Hamiltonian, which in general has the following form,

$$
H(\mathbf{k})=\sum_{i, j=0}^{3} a_{i j}(\mathbf{k}) \sigma_{i} \tau_{j}=\left(\begin{array}{ll}
h_{\uparrow \uparrow}(\mathbf{k}) & h_{\uparrow \downarrow}(\mathbf{k}) \\
h_{\downarrow \uparrow}(\mathbf{k}) & h_{\downarrow \downarrow}(\mathbf{k})
\end{array}\right),
$$

where the Pauli matrix $\sigma_{1,2,3}\left(\tau_{1,2,3}\right)$ indicates the spin (orbital) degrees of freedom and $\sigma_{0}$ and $\tau_{0}$ are the $2 \times 2$ identity matrices. $h_{\sigma \sigma^{\prime}}\left(\sigma, \sigma^{\prime}=\uparrow, \downarrow\right)$ indicates a $2 \times 2$ matrix, which can be spanned by $\tau_{0,1,2,3}$ and $a_{i j}(\mathbf{k})$ are real functions of $\mathbf{k}$. The invariance of the system under the $C_{n}$ rotation (the $n$-fold rotation about a principle axis) gives $C_{n} H(\mathbf{k}) C_{n}^{-1}=H\left(R_{n} \mathbf{k}\right)$, where $R_{n}$ is the $3 \times 3$ rotation matrix defining the $2 \pi / n$ rotation in the $3 \mathrm{D}$ space 21 . Without loss of generality, we can choose the $k_{z}$ axis as the axis of the $C_{n}$ rotation. Then along the $k_{z}$ axis on which $R_{n} \mathbf{k}=\mathbf{k}$ is satisfied, $\left[C_{n}, H(\mathbf{k})\right]=0$. Therefore, we can choose a basis in which both $\left.H\left(k_{z}\right)\right|_{k_{x}=k_{y}=0}$ and $C_{n}$ are diagonal, hence, all bands on the $k_{z}$ axis can be labelled by the corresponding eigenvalues of $C_{n}$. In such a basis, the Hamiltonian can be written as $\left.H\left(k_{z}\right)\right|_{k_{x}=k_{y}=0}=d_{0}+d_{1} \sigma_{3}+d_{2} \tau_{3} \sigma_{3}+d_{3} \tau_{3}$, where $d_{0,1,2,3}\left(k_{z}, m\right)$ are real functions. As the simultaneous presence of TRS and IS requires the double degeneracy of each state, among $d_{1,2,3}$, only one function can be non-zero. Also, as the degenerate bands should have the opposite spin directions, $d_{1}=0$. Hence, the Hamiltonian becomes $\left.H\left(k_{z}\right)\right|_{k_{x}=k_{y}=0}=d_{0}+d\left(k_{z}, m\right) \Gamma$, where $\Gamma$ is either $\Gamma=\tau_{3}$ or $\Gamma=\sigma_{3} \tau_{3}$. Then, as the energy gap is given by $2\left|d\left(k_{z}, m\right)\right|$, an ABC can be achieved if and only if $d\left(k_{z}, m\right)=0$. Here the number of variables (two) is larger than the number of equations (one) to be satisfied for the band crossing, hence, the Dirac SM can always be created via an ABC.

Moreover, owing to the TRS and IS, $d\left(k_{z}\right)$ has a definite parity under the sign reversal of $k_{z}$ as shown in Methods. In fact, the parity of $d\left(k_{z}\right)$ can be simply determined by the matrix representation $P$ of the IS. Namely, when $P$ has a diagonal form such as $P= \pm \tau_{0}$ or $\pm \tau_{z}, d\left(k_{z}\right)$ is even, whereas it is odd if $P$ has an off-diagonal form $P= \pm \tau_{x}$. At first, when $d\left(k_{z}\right)$ is even, $d\left(k_{z}\right) \approx M+\frac{1}{2} t_{z} k_{z}^{2}$ in the leading order with constants $M$ and $t_{z}$. In this case, the system is a gapped insulator (a Dirac SM) when $M t_{z}>0\left(M t_{z}<0\right)$. Namely, by taking $M$ as a tunable parameter 
a

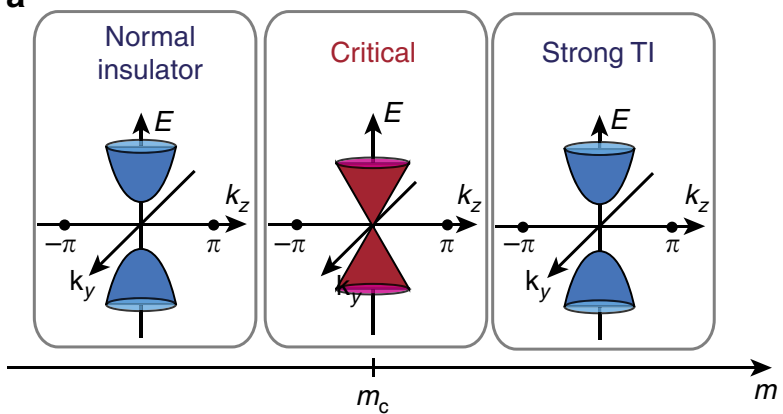

b

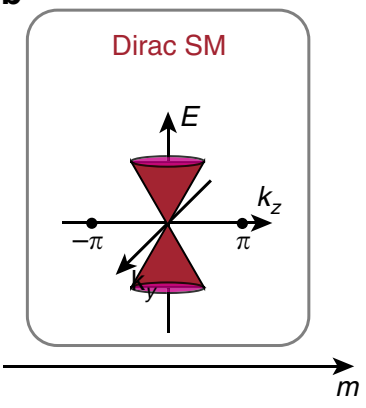

C
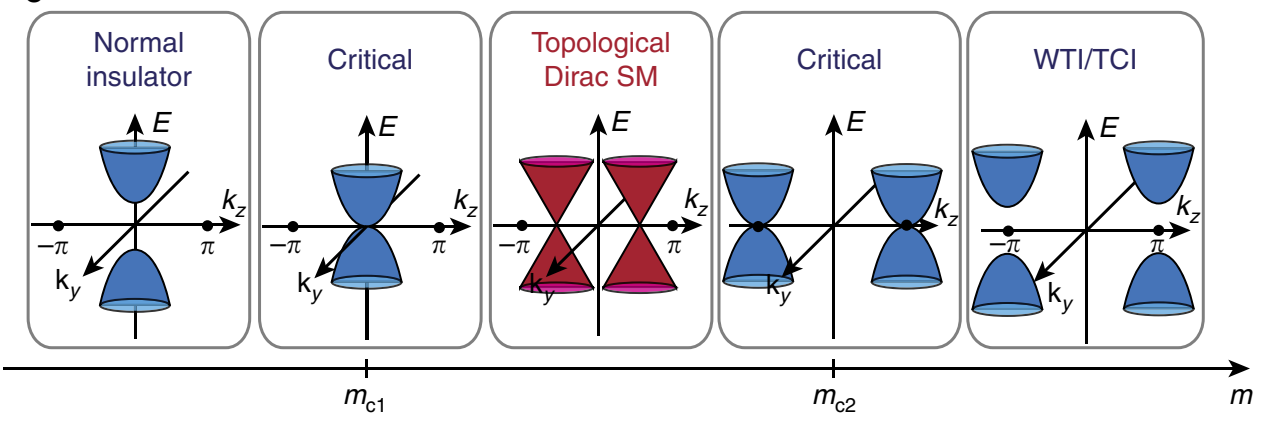

Figure 1 | Creation of a topological Dirac semimetal via accidental gap closings. (a) The phase transition induced by an accidental band crossing (ABC) when a single control parameter $m$ is varied in systems with the TRS and IS but lacking the rotation symmetry. The 3D Dirac fermion appears only at the critical point $m=m_{c}$ and the $A B C$ mediates the transition between a normal insulator and a strong topological insulator ( $\left.\mathrm{TI}\right)$. When an additional uniaxial rotational symmetry is included, two different phase diagrams can be obtained as shown in $\mathbf{b}$ and $\mathbf{c}$. Here we choose the $k_{z}$ axis as the axis for the $n$-fold rotation. (b) The Dirac SM persists irrespective of $m$. The Dirac point locates at a TRIM on the rotation axis. The Dirac SM in Table 2 corresponds to this case. (c) A stable topological Dirac SM phase appears when $m_{\mathrm{c} 1}<m<m_{\mathrm{c} 2}$, which mediates the transition between a normal insulator and a weak topological insulator (WTI) or a topological crystalline insulator ( $\mathrm{TCl}$ ). Here a pair of bulk Dirac points, each of which has fourfold degeneracy at the gapless point, exist along the rotation axis and approach the Brillouin zone (BZ) boundary as $m$ increases. The Dirac SM in Table 1 corresponds to this case. At the quantum critical points $\left(m=m_{\mathrm{c} 1}\right.$ or $m=m_{\mathrm{c} 2}$ ), the energy dispersion along the $k_{z}$ direction is quadratic while the dispersion along the $k_{\mathrm{x}}$ and $k_{\mathrm{y}}$ directions is linear (linear Dirac SM) or quadratic (quadratic Dirac SM) or cubic (cubic Dirac SM).

\section{Table 1 | Classification table for 3D topological Dirac semimetals.}

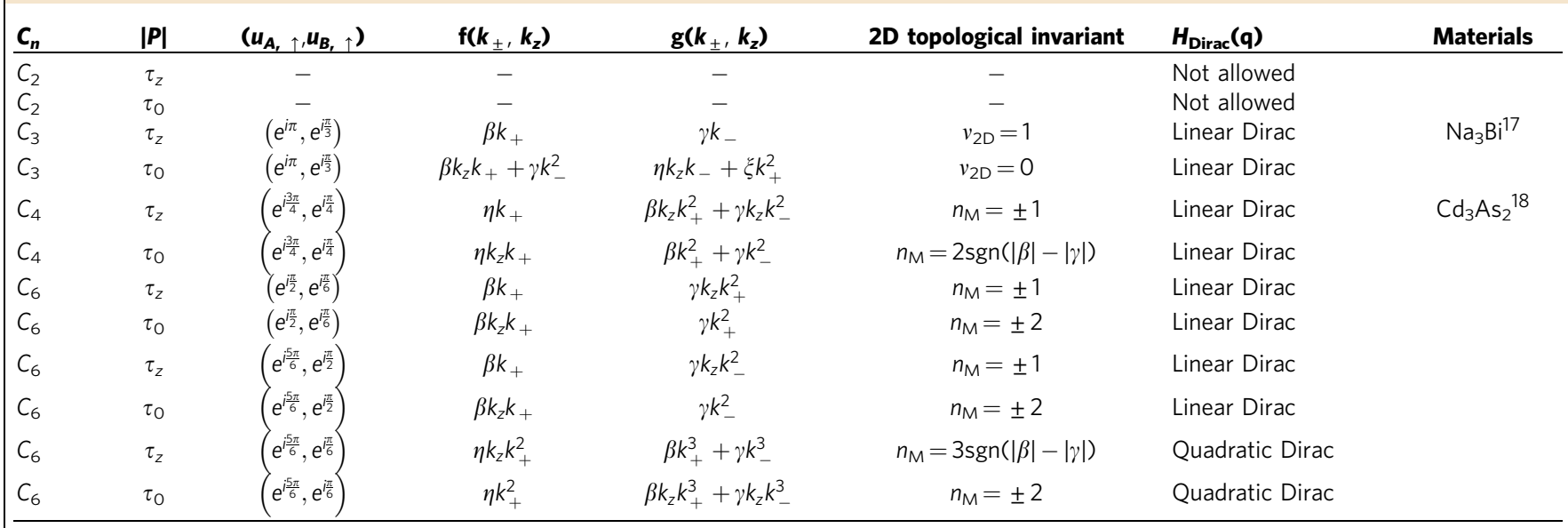

2D, two-dimensional; 3D, three-dimensional.

Classification table for 3D topological Dirac semimetals obtained by an accidental band crossing in systems having $C_{n}$ rotational symmetry with respect to the $z$ axis. Here $C_{n}=$

$\operatorname{diag}\left[u_{A, \uparrow}, u_{B, \uparrow}, u_{A, \downarrow}=u_{A \uparrow \uparrow}^{*}, u_{B, \downarrow}=u_{B, \uparrow}^{*}\right]$ and $\beta, \gamma, \eta, \xi$ are complex numbers. For compact presentation, $u_{A, \uparrow}$ and $u_{B, \uparrow}$ are arranged in a way that $0<\arg \left(u_{B, \uparrow}\right)<\arg \left(u_{A, \uparrow}\right) \leq \pi . v_{2 D}\left(n_{M}\right)$ indicates the $2 D Z_{2}$ invariant (mirror Chern number) defined on the $k_{z}=0$ plane $\left(n_{M}=v_{2 \mathrm{D}}\right.$ mod 2.). The $2 \times 2$ Hamiltonian $h_{\uparrow \uparrow}(\mathbf{k})=f(\mathbf{k}) \tau_{+}+f^{\star}(\mathbf{k}) \tau_{-}+a_{5}(\mathbf{k}) \tau_{z}$. In the case of $h_{\uparrow}(\mathbf{k}), h_{\uparrow \downarrow}(\mathbf{k})=g(\mathbf{k}) \tau_{x}$ when $P= \pm \tau_{z}$ while $h_{\uparrow l}(\mathbf{k})=g(\mathbf{k}) \tau_{y}$ when $P= \pm \tau_{0}$. The leading order terms of $f(\mathbf{k})$ and $g(\mathbf{k})$ are shown in the table. $H_{\text {Dirac }}(\mathbf{q})$ describes the effective Hamiltonian near the bulk Dirac point, which is either $h_{\uparrow \downarrow}(\mathbf{k})=g(\mathbf{k}) \tau_{y}$ when $P= \pm \tau_{0}$. The leading order terms of $f(\mathbf{k})$ and $g(\mathbf{k})$ are shown in the table. $H_{\text {Dirac }}(\mathbf{q})$ describes the effective Hamiltonian near the bulk Dirac point, which is either
$H_{\text {Dirac }}(\mathbf{q})=v_{x} q_{x} \Gamma_{1}+v_{y} q_{y} \Gamma_{2}+v_{z} q_{z} \Gamma_{3}$ (Linear Dirac) or $H_{\text {Dirac }}(\mathbf{q})=v_{x}\left(q_{x}^{2}-q_{y}^{2}\right) \Gamma_{1}+2 v_{y} q_{x} q_{y} \Gamma_{2}+v_{z} q_{z} \Gamma_{3}$ (Quadratic Dirac), where $\Gamma_{1,2,3}$ are mutually anticommuting $4 \times 4$ Gamma matrices and $v_{x, y, z}$ are real constants. Here the momentum $\mathbf{q}$ is measured with respect to the bulk Dirac point.

and assuming $t_{z}<0$, the transition from an insulator $(M<0)$ to a 3D Dirac SM $(M>0)$ can be achieved across the sign reversal of $M$ (or the band inversion). In particular, the 3D Dirac SM phase possesses two DPs, which are symmetrically located with respect to the centre of the rotation axis at $k_{z}= \pm \sqrt{2 M /\left|t_{z}\right|}$. The Dirac SMs realized in $\mathrm{Cd}_{3} \mathrm{As}_{2}$ and $\mathrm{Na}_{3} \mathrm{Bi}$ belong to this class ${ }^{17,18}$. 
On the other hand, when $d\left(k_{z}\right)$ is odd, $d\left(k_{z}\right) \approx v k_{z}$ in the leading order with a constant $v$. In this case, there is a single 3D DP at $k_{z}=0$. Then the system is nothing but a stable 3D Dirac SM with a single DP at the centre of the rotation axis. Considering the full periodic structure of the Brillouin zone (BZ), both $k_{z}=0$ and $k_{z}=\pi$, that is, the TRIMs on the rotation axis, are the possible locations of the DP. The candidate Dirac SM systems such as $\beta$-cristobalite $\mathrm{BiO}_{2}$ (ref. 15) and distorted spinels ${ }^{16}$ proposed by the recent theoretical studies belong to this class. In these systems, $P= \pm \tau_{x}$ representation is realized due to the non-symmorphic space group symmetry of the system with two sublattice degrees of freedom. Namely, the system is invariant under the inversion with respect to a lattice site followed by a partial translation between sublattice sites. As the sublattices are interchanged through the symmetry operation, $P$ can have an off-diagonal form of $P= \pm \tau_{x}$. In distorted spinels, the compound IS combined with additional twofold rotation and time-reversal symmetries gives rise to a single $\mathrm{DP}$ at the $T$ point of the $\mathrm{BZ}^{16}$. On the other hand, there are three bulk DPs in $\beta$-cristobalite $\mathrm{BiO}_{2}$ at the three symmetry-related $X$ points ${ }^{15}$. At each $X$ point, the system has a uniaxial fourfold rotation symmetry with the compound IS $P$ consistent with our theory.

In conclusion, when the material has the TRS, IS and uniaxial rotation symmetry simultaneously, there are two different ways to obtain a stable Dirac SM phase. One is through an ABC (or a band inversion), which gives rise to a stable 3D Dirac SM with a pair of 3D DPs on the axis of the rotation (see Fig. 1c). This class of Dirac SMs appears when the IS is represented by $P= \pm \tau_{0}$ or $\pm \tau_{z}$. The other case is when the system naturally supports a $3 \mathrm{D}$ $\mathrm{DP}$ at a TRIM on the rotation axis due to the symmetry of the system (see Fig. 1b). Such a Dirac SM can exist when the IS is represented by $P= \pm \tau_{x}$. The intrinsic properties of the relevant 3D Dirac SM phases are summarized in Tables 1 and 2, respectively. Up to now, we have considered only the leading order terms of $d\left(k_{z}\right)$. However, when the higher order terms are included, the number of DPs can be increased as a pair of DPs can always be created whenever an additional ABC happens. However, independent of the total number of DPs, the Dirac SM with $P= \pm \tau_{0, z}$ always has an even number of DPs away from the TRIM, whereas the Dirac SM with $P= \pm \tau_{x}$ having an odd number of DPs always possesses a single DP at a TRIM.

Classification table. The classification of the 3D Dirac SM phases can be performed rigorously by imposing the TRS, IS and $C_{n}$ rotation symmetry to the minimal $4 \times 4$ Hamiltonian $H(\mathbf{k})$. In the simultaneous presence of the TRS and IS, the Hamiltonian can always be written as $H(\mathbf{k})=\sum_{i=1}^{5} a_{i}(\mathbf{k}) \Gamma_{i}$, where $\Gamma_{i}$ indicates
$4 \times 4$ hermitian matrices satisfying $\left\{\Gamma_{i}, \Gamma_{j}\right\}=2 \delta_{i j}$, which guarantees the double degeneracy of eigenstates at each $\mathbf{k}$ (see Methods). The precise functional form of $a_{i}(\mathbf{k})$ can be fixed by imposing the $C_{n}$ rotational symmetry using the basis under which both $C_{n}$ and $H(\mathbf{k})$ are diagonal along the $k_{z}$ direction ${ }^{21}$. The results of the complete classification are concisely summarized in Tables 1 and 2. Because of the TRS, the rotation operator becomes $C_{n}=\operatorname{diag}\left[u_{A, \uparrow}, u_{B, \uparrow}, u_{A, \uparrow}^{*}, u_{B, \uparrow}^{*}\right]$, where each component $u$ can be written as $u=\exp \left[i \frac{2 \pi}{n}\left(p+\frac{1}{2}\right)\right]$ with $p=0,1, \ldots, n-1$ and $A, B$ indicate the orbital degrees of freedom. Hence, only two components of $C_{n}$ are independent.

The properties of the 3D Dirac SM generated via ABCs are shown in Table 1 . In general, the $\mathrm{ABC}$ requires the valence and conduction bands to have different $C_{n}$ eigenvalues. Namely, $\left\{u_{A, \uparrow}\right.$, $\left.u_{A, \downarrow}\right\}$ and $\left\{u_{B, \uparrow}, u_{B, \downarrow}\right\}$ should not have common elements to avoid interband hybridization. However, in the case of $C_{2}$ invariant systems, $\left\{u_{A, \uparrow}, u_{A, \downarrow}\right\}=\left\{u_{B, \uparrow}, u_{B, \downarrow}\right\}=\{i,-i\}$, hence, the ABC is not allowed. On the other hand, the systems with $C_{3}, C_{4}$ and $C_{6}$ symmetries can support 3D Dirac SM phases. Although the detailed structure of the Hamiltonian $H(\mathbf{k})$ depends on the specific symmetries of the corresponding system, there are only two different types of low-energy effective Hamiltonians near the bulk gap-closing point. In the first case, after some suitable unitary transformations, the effective Hamiltonian near each of the bulk DP can be written as

$$
H_{\text {Dirac }}(\mathbf{q}) \sim\left(\begin{array}{cc}
q_{x} \tau_{x}+q_{y} \tau_{y}+q_{z} \tau_{z} & 0 \\
0 & -q_{x} \tau_{x}-q_{y} \tau_{y}-q_{z} \tau_{z}
\end{array}\right),
$$

where the momentum $\mathbf{q}$ is measured with respect to the DP. This is the Hamiltonian for the conventional Dirac fermion (the linear Dirac fermion), which is composed of two Weyl fermions having the Chern number +1 or -1 , respectively. Whereas in the case of the $C_{6}$ invariant system with the $\left\{u_{A, \uparrow}, u_{B, \uparrow}\right\}=\left\{e^{i \frac{5 \pi}{6}}, e^{i \frac{\pi}{6}}\right\}$, the effective Hamiltonian is given by

$$
\begin{aligned}
& H_{\text {Dirac }}(\mathbf{q}) \sim \\
& \left(\begin{array}{cc}
\left(q_{x}^{2}-q_{y}^{2}\right) \tau_{x}+2 q_{x} q_{y} \tau_{y}+q_{z} \tau_{z} & 0 \\
0 & -\left(q_{x}^{2}-q_{y}^{2}\right) \tau_{x}-2 q_{x} q_{y} \tau_{y}-q_{z} \tau_{z}
\end{array}\right)
\end{aligned}
$$

Note that this DP is composed of two double-Weyl fermions, which have the Chern number +2 or -2 , respectively ${ }^{21,22}$. Hence, we call this gapless fermions as the quadratic Dirac fermions.

The physical properties of the 3D Dirac SM with a DP at the centre of the rotation axis are summarized in Table 2 . As the IS

\begin{tabular}{|c|c|c|c|c|c|c|}
\hline$c_{n}$ & $|\boldsymbol{P}|$ & $\boldsymbol{u}_{\mathbf{A}, \uparrow}$ & $f\left(k_{ \pm}, k_{z}\right)$ & $g_{z}\left(k_{ \pm}, k_{z}\right)$ & $H_{\text {Dirac }}(q)$ & Material \\
\hline$\overline{C_{2}}$ & $\tau_{x}$ & $e^{-\frac{\pi}{2}}$ & $k_{z} F_{1}^{(1)}\left(k_{x, y}\right)-i F_{2}^{(1)}\left(k_{x, y}\right)$ & $\alpha k_{x}+\beta k_{y}$ & Linear Dirac & Distorted spinels $^{16}$ \\
\hline $\mathrm{C}_{4}$ & $\tau_{x}$ & $e^{ \pm \frac{\pi}{4}}$ & $F_{1}^{(2)}\left(k_{x, y}\right)-i k_{z} F_{2}^{(2)}\left(k_{x, y}\right)$ & $\alpha k_{ \pm}$ & Linear Dirac & $\mathrm{BiO}_{2}^{15}$ \\
\hline$C_{6}$ & $\tau_{x}$ & $e^{ \pm \frac{\pi}{6}}$ & $k_{z} F_{1}^{(3)}\left(k_{x, y}\right)+i F_{2}^{(3)}\left(k_{x, y}\right)$ & $\alpha k_{ \pm}$ & Linear Dirac & \\
\hline
\end{tabular}
flips the orbitals in this case $\left(P= \pm \tau_{x}\right)$, the doubly degenerate states at each momentum on the $k_{z}$ axis have different orbitals

3D, three-dimensional; SM, semimetal.

Classification table for $3 \mathrm{D}$ topological Dirac SMs in systems having $C_{n}$ rotational symmetry with respect to the $z$ axis when $P= \pm \tau_{x}$. In this Dirac SM phase, the location of the 3D Dirac point is fixed either at the centre or the edge of the rotation axis, that is, at a time-reversal invariant momentum on the rotation axe. Here $C_{n}=\operatorname{diag}\left[u_{A, \uparrow}, u_{B, \uparrow}, u_{A, 1}, u_{B, 1}\right]=\operatorname{diag}\left[u_{A, \uparrow},-u_{A, \uparrow}, u_{A, \uparrow}^{*},-u_{A, \uparrow}^{*}\right]$ and $\alpha, \beta$ are complex numbers. For compact presentation, $\arg \left(u_{A, \uparrow}\right)$ is fixed to be $-\frac{\pi}{2} \leq \arg \left(u_{A, \uparrow}\right) \leq \frac{\pi}{2}$. But the same result holds even if arg $\left(u_{A, \uparrow}\right)$ is shifted by $\pi$. The real functions $F^{(1,2,3)}$ are given by complex numbers. For compact presentation, $\arg \left(u_{A, \uparrow}\right)$ is fixed to be $-\frac{\pi}{2} \leq \arg \left(u_{A, \uparrow}\right) \leq \frac{\pi}{2}$. But the same result holds even if arg $\left(u_{A, \uparrow}\right)$ is shifted by $\pi$. The real functions $F^{(1,2,3)}$ are given by
$F_{i=1,2}^{(1)}=c_{i}^{(1)} k_{x}+d_{i}^{(1)} k_{y}, F_{i=1,2}^{(2)}=c_{i}^{(2)}\left(k_{x}^{2}+k_{y}^{2}\right)+d_{i}^{(2)} k_{x} k_{y} F_{i=1,2,3,4}^{(3)}=c_{i}^{(3)}\left(k_{+}^{3}+k_{-}^{3}\right)+i d_{i}^{(3)} k_{+}^{3}-k_{-}^{3}$ where $c_{i}^{(1,2,3)}$ and $d_{i}^{(1,2,3)}$ are real constants. The $2 \times 2$ Hamiltonian $h_{\uparrow \uparrow}(\mathbf{k})=f_{(\mathbf{k})}(\mathbf{k})+f_{+}^{*}(\mathbf{k}) \tau_{-}+a_{1}(\mathbf{k}) \tau_{z}$ where $a_{1}(\mathbf{k})=v k_{z}$ with a real constant $v$, and $h_{\uparrow}(\mathbf{k})=g_{z}(\mathbf{k}) \tau_{z}$. The leading order terms of $f(\mathbf{k})$ and $g_{z}(\mathbf{k})$ are shown in the table. H which is either $H_{\text {Dirac }}(\mathbf{q})=v_{x} q_{x} \Gamma_{1}+v_{y} q_{y} \Gamma_{2}+v_{z} q_{z} \Gamma_{3}$ (Linear Dirac) or $H_{\text {Dirac }}(\mathbf{q})=v_{x}\left(q_{+}^{3}+q_{-}^{3}\right) \Gamma_{1}+i v_{y}\left(q_{+}^{3}-q_{-}^{3}\right) \Gamma_{2}+v_{z} q_{z} \Gamma_{3}$ (Cubic Dirac), where the momentum $\mathbf{q}$ is measured with respect to the bulk Dirac point with $q_{ \pm}=q_{x} \pm i q_{y}$. Here $\Gamma_{1,2,3}$ are mutually anticommuting $4 \times 4$ Gamma matrices and $v_{x, y, z}$ are real constants. 
and opposite spin directions. Therefore, the band crossing between degenerate bands requires $\left\{u_{A, \uparrow}, u_{B, \downarrow}\right\} \cap\left\{u_{B, \uparrow}, u_{A, \downarrow}\right\}=\emptyset$ contrary to the previous case. Moreover, owing to the additional constraint of $u_{A, \uparrow}=-u_{B, \uparrow}$, the Dirac SM phase with a single DP cannot exist in the system with $C_{3}$ invariance, whereas the systems with $C_{2}, C_{4}, C_{6}$ symmetries support it (see Methods). One interesting prediction of Table 2 is that when the system has $C_{6}$ symmetry with $u_{A, \uparrow}= \pm e^{\frac{3 \pi}{6}}$, the low-energy Hamiltonian near the DP can be written as

$H_{\text {Dirac }}(\mathbf{q}) \sim$

$\left(\begin{array}{cc}\left(q_{+}^{3}+q_{-}^{3}\right) \tau_{x}+i\left(q_{+}^{3}-q_{-}^{3}\right) \tau_{y}+q_{z} \tau_{z} & 0 \\ 0 & -\left(q_{+}^{3}+q_{-}^{3}\right) \tau_{x}-i\left(q_{+}^{3}-q_{-}^{3}\right) \tau_{y}-q_{z} \tau_{z}\end{array}\right)$.

Note that this DP is composed of two triple-Weyl fermions, which have the Chern number +3 or -3 , respectively ${ }^{21}$. Hence, we can call this gapless fermion as the cubic Dirac fermion. For the other cases in Table 2, the effective Hamiltonian near the DP is simply described by the ordinary linear Dirac fermions.

Topological properties of the 3D Dirac semimetals. One important characteristic of the 3D Dirac SM generated by an $\mathrm{ABC}$ is that it carries a quantized topological invariant although it is a gapless SM. In fact, the band inversion associated with the $A B C$ is the common origin of the presence of the $2 \mathrm{D}$ topological invariant and the emergence of bulk DPs. As shown in Table 1, in each case, a quantized $2 \mathrm{D}$ topological invariant can be defined on the $k_{z}=0$ plane where the $\mathrm{ABC}$ occurs. First of all, as the $k_{z}=0$ plane can be considered as a 2D system with TRS, a $2 \mathrm{D} Z_{2}$ invariant $v_{2 \mathrm{D}}$ is well-defined on $\mathrm{it}^{23,24}$. Moreover, because of the simultaneous presence of the TRS and IS, $v_{2 \mathrm{D}}$ can be determined by the parities of the occupied bands at TRIM ${ }^{25}$. Therefore, when the valence and conduction bands have the opposite parities $\left(P= \pm \tau_{z}\right)$, the band inversion on the $k_{z}=0$ plane, which generates a pair of bulk DPs, changes $v_{2 \mathrm{D}}$ by 1 , that is, $\Delta v_{2 \mathrm{D}}=1$. This can be contrasted to the case when two bands have the same parity $\left(P= \pm \tau_{0}\right)$, in which $\Delta v_{2 \mathrm{D}}=0$.

On the other hand, the presence of additional crystalline symmetries allows us to introduce new topological invariants ${ }^{26-28}$. In particular, when the system has either $C_{4}$ or $C_{6}$ symmetry, the $k_{z}=0$ plane carries an integer topological invariant (the mirror Chern number) because of the mirror symmetry of the system $^{29,30}$. Here the mirror symmetry appears because of the simultaneous presence of the $\pi$ rotation $\left(R_{\pi}\right)$ with respect to the $k_{z}$ axis and the IS. Then, the combined operation of the IS and $\pi$ rotation defines the mirror symmetry $M=P R_{\pi}$, which connects the Hamiltonian $H\left(k_{x}, k_{y}, k_{z}\right)$ and $H\left(k_{x}, k_{y},-k_{z}\right)$, hence, the system is invariant under the mirror symmetry $(M)$ in the $k_{z}=0$ plane. As $M^{2}=-1$, the Hamiltonian can be block-diagonalized with each block characterized by the mirror eigenvalue $\pm i$, respectively. Then, the Chern number can be defined in each block $\left(C_{+i}\right)$, separately. Although the total Chern number $C_{+i}+C_{-i}=0$ because of the TRS, the difference $n_{\mathrm{M}} \equiv$ $\frac{1}{2}\left(C_{i}-C_{-i}\right)$ (the mirror Chern number) can be non-zero. Note that $R_{\pi}$ exists only in systems with the $C_{4}$ or $C_{6}$ symmetry with the corresponding $R_{\pi}=C_{4}^{2}$ or $R_{\pi}=C_{6}^{3}$, respectively. Therefore, the $C_{3}$ invariant system is characterized only by the $2 \mathrm{D} Z_{2}$ invariant $v_{2 \mathrm{D}}$, whereas the system with the $C_{4}$ or $C_{6}$ symmetry has both the $Z_{2}$ invariant $v_{2 \mathrm{D}}$ and the mirror Chern number $n_{\mathrm{M}}$, where $n_{\mathrm{M}}$ and $v_{2 \mathrm{D}}$ are equivalent up to the modulo 2. In Methods, we have described how the mirror symmetry manifests in the system in terms of the effective Hamiltonian $H(\mathbf{k})$. When either $v_{2 \mathrm{D}}$ or $n_{\mathrm{M}}$ is non-zero, the 3D Dirac SM supports 2D surface Dirac cones when a surface parallel to the $k_{z}$ axis is introduced. The number of 2D Dirac cones on one surface is given by $\left|n_{\mathrm{M}}\right|$ $\left(\left|v_{2 \mathrm{D}}\right|\right)$ when the system has the $C_{4}$ or $C_{6}\left(C_{3}\right)$ symmetry.
Lattice model and generic phase diagram. Let us illustrate the intriguing properties of the $3 \mathrm{D}$ topological Dirac SM phases in Table 1 by studying lattice Hamiltonians numerically. For convenience, we choose the $C_{4}$ invariant systems with $P=\tau_{0}$ or $P=\tau_{z}$ corresponding to the 6th or 7 th row of Table 1 , respectively. However, the main features of the phase diagram can be applied to all cases in Table 1 because the overall structure of the phase diagram is solely determined by the single function $a_{5}(\mathbf{k})$, whose leading order functional form is the same in all cases. The detailed information about the lattice Hamiltonian is presented in Methods. Figure 2 summarizes the main properties of the lattice Hamiltonian. In general, the system supports four different phases as shown in the phase diagram. The phase transition is always accompanied by an $\mathrm{ABC}$ on the $k_{z}=0$ or $k_{z}=\pi$ plane, in which a pair of 3D bulk DPs are either created or annihilated. Whenever an $\mathrm{ABC}$ happens, it also changes the 2D topological invariant of the corresponding $2 \mathrm{D}$ planes.

There are two different types of insulators in the phase diagram. One is a normal insulator, which does not carry a topological number, and the other is either a weak topological insulator or a topological crystalline insulator, in which both the $k_{z}=0$ and $k_{z}=\pi$ planes have nontrivial 2D topological invariants. The system with $P= \pm \tau_{z}$ supports a weak topological insulator, which has the $Z_{2}$ topological index of $\left(v_{0} ; v_{1} v_{2} v_{3}\right)=(0 ; 001)$ because $v_{2 \mathrm{D}}=1$ in both the $k_{z}=0$ and $k_{z}=\pi$ planes. On the other hand, when $P= \pm \tau_{0}, v_{0,1,2,3}=0$ independent of the band inversion because every band has the same parity. However, it is possible to obtain a topological crystalline insulator, as both the $k_{z}=0$ and $k_{z}=\pi$ planes can have the non-zero mirror Chern number of $n_{\mathrm{M}}=2$. In fact, it is to note that the weak topological insulator in the system with $P= \pm \tau_{z}$ can also have finite mirror Chern numbers. However, for convenience, we mainly focus on the $Z_{2}$ invariant to characterize the system with $P= \pm \tau_{z}$.

The 3D Dirac SM phases can also be distinguished in two different ways. At first, when the Dirac SM phase has a non-zero topological invariant in either the $k_{z}=0$ plane or the $k_{z}=\pi$ plane, we can call it a topological Dirac SM as the Dirac SM carries stable 2D Dirac cones on the surface. Similarly, a topologically trivial Dirac SM can be defined when the system does not have any topological invariant in both planes. However, in both cases, independent of the presence of the $2 \mathrm{D}$ topological invariants, the 3D Dirac SM phase is stable because the symmetry of the system and occupies a finite region in the phase diagram.

Figure 3 shows the evolution of the Fermi surface of the topological Dirac SM system with a slab geometry whose surface normal is parallel to the [100] direction. The translational symmetry of the system in the $y z$ plane is maintained. Here we first pick the states touching the Fermi level $\left(E_{\mathrm{F}}\right)$ and then plot the wave function amplitudes of the corresponding state localized on the first five layers from the top surface. The red colour indicates the states localized on the [100] surface, whereas the bright blue colour corresponds to the 3D bulk Dirac states. In the case of the topological Dirac SM with $v_{2 \mathrm{D}}=1$ in the $k_{z}=0$ plane, a $2 \mathrm{D}$ surface Dirac cone appears on the $k_{y}$ axis centred at the $\Gamma$ point as shown in Fig. 3a. Here the two bulk DPs give rise to the finite intensity on the $k_{z}$ axis located symmetrically with respect to the $\Gamma$ point. When the Fermi energy $\left(E_{\mathrm{F}}\right)$ is near the bulk DP $\left(E_{\mathrm{F}}=0\right.$ at the DP), the bulk and surface states are decoupled, and the surface states form an isolated closed loop. As $E_{\mathrm{F}}$ increases, the Fermi surface topology evolves continuously, and when the bulk and surface states start to overlap, the $2 \mathrm{D}$ surface state is deformed to the Fermi arc structure. Namely, the Fermi arcs of 3D Dirac SM emerge simply because of the deformation of the 2D Dirac cone, which exists due to the fact that $v_{2 \mathrm{D}}=1$ on the $k_{z}=0$ plane. As such an evolution of the surface spectrum occurs 


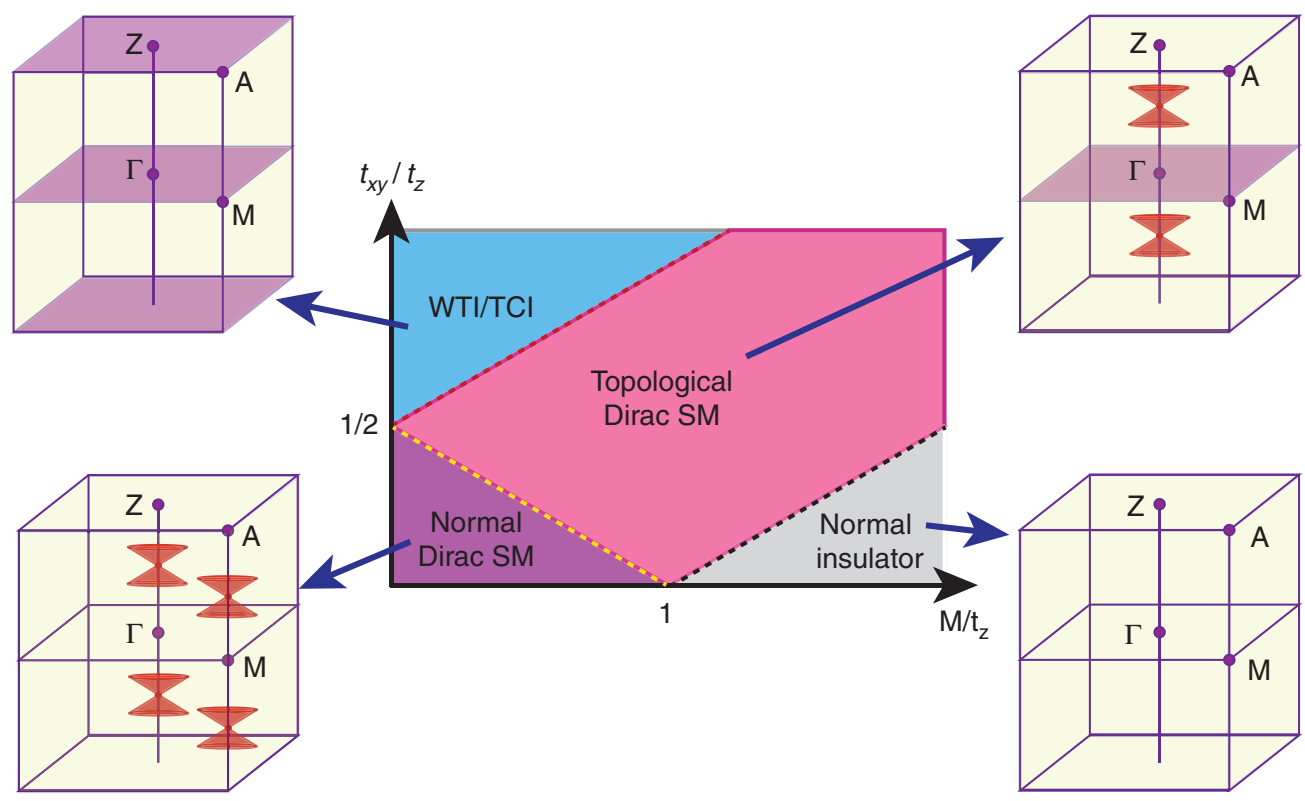

Figure 2 | Phase diagram. Generic phase diagram of the system with the time-reversal, inversion and rotation symmetries. It is obtained by numerically solving the lattice Hamiltonians in equations (16) and (19), both of which lead to the same phase diagram. Here $M$ indicates the on-site energy difference between two orbitals and $t_{x y}\left(t_{z}\right)$ describes the hopping amplitude along the direction perpendicular (parallel) to the rotation axis. For each phase, the location of the 3D bulk Dirac point in the first $B Z$, if it is present, is indicated by the red symbol having the shape of a Dirac cone. When the two-dimensional (2D) plane with $k_{z}=0$ or $k_{z}=\pi$ possesses a nontrivial 2D topological invariant, such as $Z_{2}$ invariant $\left(v_{2 D}\right)$ or the mirror Chern number $\left(n_{M}\right)$, the corresponding plane is coloured in purple. If any of these 2D planes carries a non-zero 2D topological invariant, the surface of the material, which is parallel to the axis of the rotation, supports 2D surface Dirac cones. In the figure, WTI and TCl indicate a weak topological insulator and a topological crystalline insulator, respectively. A gap-closing happens at the $\Gamma(Z)$ point on the black (red) dotted line, whereas a gap-closing occurs at the $M$ point on the yellow dotted line.

continuously, the Fermi arc states can appear even when $E_{\mathrm{F}}=0$ if the parameters of the model Hamiltonian are tuned properly.

The energy spectrum of the topological Dirac SM with $n_{\mathrm{M}}=2$ on the $k_{z}=0$ plane also shows a similar variation as described in Fig. 3b. As $n_{\mathrm{M}}=2$, there are two 2D surface Dirac cones on the $k_{y}$ axis. As $E_{\mathrm{F}}$ increases, the surface Dirac cones evolve to Fermi arcs when the surface and bulk states overlap. As the number of surface Dirac cones is two, the number of surface Fermi arc is also doubled as compared with the case shown in Fig. 3a. It is worth to note that in both cases shown in Fig. 3a,b, the energy dispersion near the bulk 3D DP is basically the same, that is, the bulk state shows the linear dispersion relation in all three momentum directions. This clearly shows that the number of Fermi arcs of the topological Dirac SM is irrespective of the dispersion of the bulk states.

It is worth to stress that the physical origin of the surface Fermi arcs in the 3D Dirac SM is clearly distinct from that of the Weyl SM which has twofold degeneracy at the gap-closing point. In the Weyl SM, the Chern number carried by the bulk gapless point (Weyl point) guarantees the emergence and stability of the Fermi arc states ${ }^{31-33}$. As the Chern number of the Weyl point is purely determined by the energy dispersion around the Weyl point, the number of Fermi arcs in the system with a fixed number of Weyl points strongly depends on the energy dispersion near the Weyl point. In conventional Weyl SMs with the linear dispersion around the Weyl point, the number of Fermi arcs on one surface of the sample is equal to the number of Weyl point pairs in the first BZ. On the other hand, in the case of Weyl SMs with double (triple)-Weyl fermions whose dispersion is quadratic (cubic) along the two momentum directions but linear in the third direction, the number of Fermi arcs is double (triple) of the number of the Weyl point pairs ${ }^{21,22}$. However, in contrast to the case of the Weyl SM, the physical origin of the surface states of the 3D Dirac SM is independent of the energy dispersion of the
3D bulk Dirac fermions. Because of the simultaneous presence of the TRS and IS, the Chern number of each 3D DP is zero. Here the number of the Fermi arcs on the surface of the sample is solely determined by the $2 \mathrm{D}$ topological invariant on the $k_{z}=0$ (or $k_{z}=\pi$ ) plane irrespective of the energy dispersion around the 3D DP. Therefore, although the low-energy Hamiltonian near the $3 \mathrm{D}$ bulk DP is the same, the number of Fermi arcs can be different depending on the $2 \mathrm{D}$ topological invariant of the system as shown above.

\section{Discussion}

Up to now, we have considered the system with a uniaxial rotation symmetry together with TRS and IS. As most crystals have a larger symmetry with multiple rotation axes, it is important to understand how our theory can be applied to these systems. First of all, it is worth to note that the location of the two DPs can be anywhere on the rotation axis in the case of the Dirac SMs with $P= \pm \tau_{0, z}$ in Table 1 . Therefore, the primary rotation axis should be the line connecting the two DPs in this case. Even if there are multiple pairs of DPs in the BZ, we can easily specify the primary rotation axis for each pair of DPs by using the fact that the centre of the rotation axis locates at a TRIM and the relative orientation of different rotation axes should satisfy the crystalline symmetry. Once the primary rotation axis is specified, we can apply our classification scheme to each pair of DPs. On the other hand, in the case of the Dirac SM with $P= \pm \tau_{x}$ in Table 2, the role of the additional crystalline symmetry is more significant because the DP locates at a TRIM where the system generally has a higher crystalline symmetry. At this point, it is useful to note that the threefold rotation symmetry prohibits a DP at a TRIM according to Table 2. In fact, this imposes a strong constraint to the allowed position of the DP, and we can rule out the $\Gamma$ point as a possible location of the DP because the space 


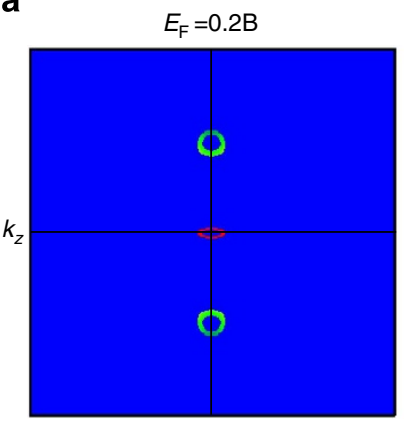

$k_{y}$

b

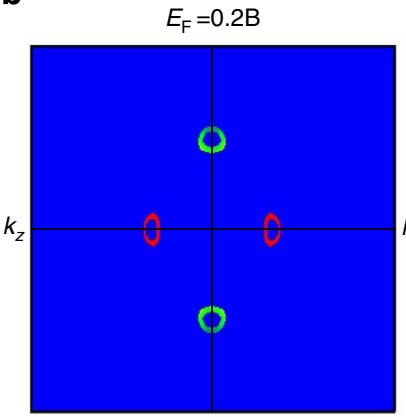

$k_{y}$

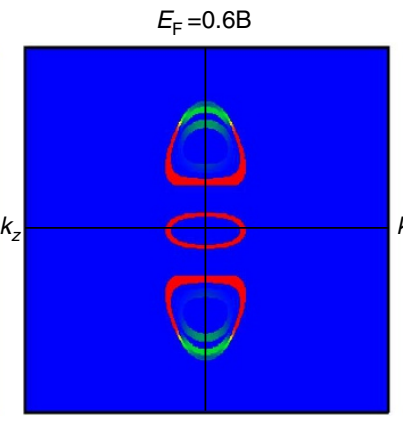

$k_{y}$

$E_{\mathrm{F}}=0.4 \mathrm{~B}$

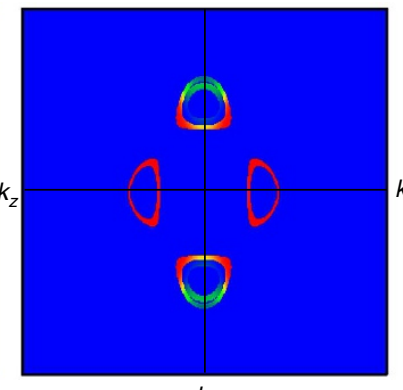

$k_{y}$

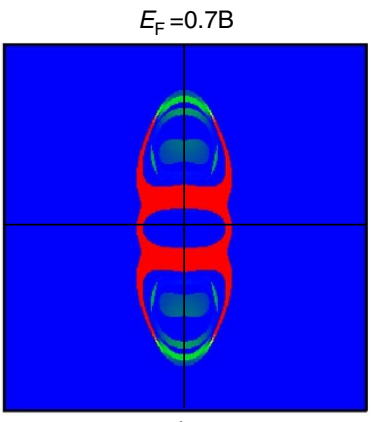

$k_{y}$

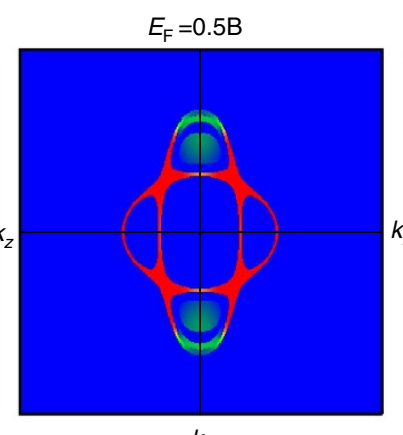

$k_{y}$

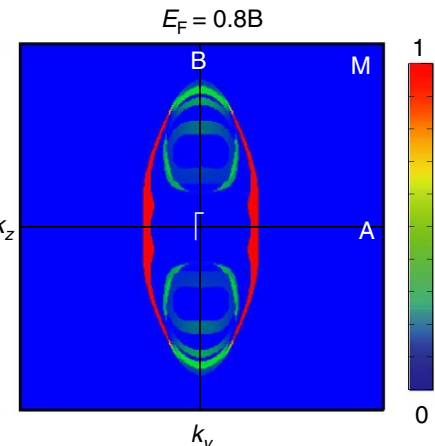

$E_{\mathrm{F}}=0.6 \mathrm{~B}$

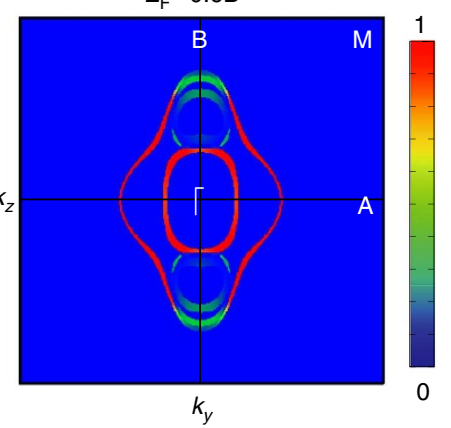

Figure 3 | Evolution of the Fermi surface of a [100] film varying the Fermi energy. The wave function amplitudes confined within the first five layers from the top surface are plotted for the states touching the Fermi level $\left(E_{\mathrm{F}}\right)$. To obtain the Fermi surface we have solved numerically the lattice Hamiltonian in equations (16) and (19). (a) For a topological Dirac semimetal with $C_{4}$ symmetry, which has $v_{2 D}=1$ on the $k_{z}=0$ plane. The closed loop at the centre of the surface BZ is from a 2D surface Dirac point at the $\Gamma$ point. Two 3D bulk Dirac cones also produce finite intensity symmetrically on the $k_{z}$ axis. As $E_{F}$ increases, the closed loop due to the 2D Dirac point deforms to a pair of Fermi arcs connected to the bulk states. (b) For a topological

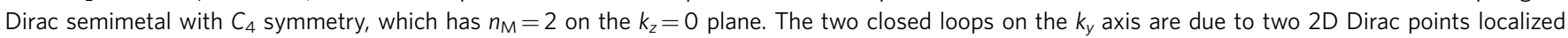
on the surface. As $E_{F}$ increases, the closed loops due to 2D Dirac cones turn into four Fermi arcs. In both a and $\mathbf{b}$, the 3D bulk Dirac fermions show the linear dispersion in the momentum space. Hence, the number of surface Fermi arcs is solely determined by the 2D topological invariant on the $k_{z}=0$ plane independent of the dispersion of the bulk Dirac fermions.

groups allowing the fourfold degeneracy at the $\Gamma$ point always have a threefold rotation symmetry (see Methods and also ref. 15). Therefore, a DP can locate only at a TRIM on the BZ surface. Even if the point group symmetry of the crystal contains many rotation axes, the symmetry on the BZ surface can be much lower and the system often has a uniaxial rotation symmetry at a TRIM on the $\mathrm{BZ}$ surface. For example, in both $\beta$-cristobalit $\mathrm{BiO}_{2}$ and distorted spinels, there is a uniaxial rotation symmetry at the momentum where each DP presents. In this situation, it is straightforward to apply our theory. To sum up, although the point group symmetry of the crystal has multiple rotation axes, the local symmetry at the momentum where each DP presents is much lower, hence, our theory can be applied to crystalline solids in general.

We conclude with a discussion about the stability of the $3 \mathrm{D}$ Dirac SM under the influence of the Coulomb interaction and disorder. Simple power counting shows that the long-range Coulomb interaction is a marginally irrelevant perturbation to the 3D Dirac fermions with the linear dispersion ${ }^{34-36}$. Hence, various physical properties of the 3D Dirac SM can receive logarithmic corrections because of the long-range Coulomb interaction similar to the cases of 3D Weyl SM ${ }^{35-37}$ and graphene ${ }^{38-40}$. On the other hand, as the disorder is irrelevant according to the power counting, we expect the Dirac SM state can be stable at least against weak disorder effect. However, as the crystalline symmetry is important for the protection of the DP, strong disorder can induce nontrivial physical consequences to 3D Dirac SM phase, especially when the interaction and disorder effect are considered simultaneously ${ }^{34}$. Moreover, in the case of the quadratic Dirac SM and the cubic Dirac SM, the effect of the interaction and disorder can be more significant. As the in-plane dispersion becomes either quadratic or cubic in the momentum space, which strongly enhances the low energy density of states, it is expected that the interaction and disorder can even bring about new exotic quantum phases. For instance, according to a recent theoretical study, an exotic non-Fermi liquid state can appear in a 3D SM having quadratic energy dispersion in the momentum space $^{41}$. As the interplay between the long-range Coulomb interaction and nontrivial screening because of the enhanced low energy density of states is the fundamental origin leading to the non-Fermi liquid phase, the quadratic Dirac SM and the cubic Dirac SM are also promising systems to observe novel quantum critical states.

Finally, let us note that the quantum critical point in Fig. 1c where the pair creation or pair annihilation of bulk DPs happens is another interesting venue to observe a new types of quantum critical phenomena. At the quantum critical point, as the energy dispersion along the rotation axis is always quadratic, the lowenergy excitation can show highly anisotropic dispersion relations in the linear Dirac SM and cubic Dirac SM. According to the recent theoretical study ${ }^{42,43}$, it is shown that such an anisotropic dispersion can induce a novel screening phenomenon, which can induce anomalous distribution of the screening charge around a charged impurity. To reveal the fascinating physical properties of the linear Dirac SM and the triple Dirac SM at the critical point would be another interesting topic for future studies. 


\section{Methods}

The classification procedure. The classification of the minimal $4 \times 4$ matrix Hamiltonian $H(\mathbf{k})$ can be performed as follows. At first, let us impose the TRS on $H(\mathbf{k})$. The TRS can be represented by the operator $\Theta=i \sigma_{y} K$, where $\sigma_{x, y, z}$ are Pauli matrices for spin degrees of freedom and $K$ stands for complex conjugation. The invariance of the Hamiltonian under TRS, that is, $H(-\mathbf{k})=\Theta H(\mathbf{k}) \Theta^{-1}$ gives rise to the relations $h_{\uparrow \uparrow}(\mathbf{k})=h_{\downarrow \downarrow}^{T}(-\mathbf{k})$ and $h_{\uparrow \downarrow}(\mathbf{k})=-h_{\uparrow \downarrow}^{T}(-\mathbf{k})$, where the superscript $T$ indicates the transposition. Then, the resulting Hamiltonian with the TRS can be written in the following way.

$$
H(\mathbf{k})=\left(\begin{array}{cc}
h_{\uparrow \uparrow}(\mathbf{k}) & h_{\uparrow \downarrow}(\mathbf{k}) \\
-h_{\uparrow \downarrow}^{*}(-\mathbf{k}) & h_{\uparrow \uparrow}^{*}(-\mathbf{k})
\end{array}\right),
$$

where the superscript ${ }^{*}$ indicates the complex conjugation. Second, to impose the IS on the Hamiltonian $H(\mathbf{k})$, we have to determine the matrix representation $P$ of the IS. As the IS is independent of the spin-rotation, in general, $P=p_{0} \tau_{0}+\vec{p} \cdot \vec{\tau}$, where $\vec{\tau}=\left(\tau_{x}, \tau_{y}, \tau_{z}\right)$ indicate the Pauli matrices for orbital degrees of freedom and $p_{0, x, y}$ are complex numbers. As the operation of $P^{2}$ relates the same electronic states, it should be equivalent to the identity operator up to a global $\mathrm{U}(1)$ phase factor, that is, $P^{2}=p_{0}^{2}+\vec{p} \cdot \vec{p}+2 p_{0} \vec{p} \cdot \vec{\tau}=e^{i 2 \phi}$. Therefore, $P$ should be either $P= \pm e^{i \phi}$ or $P=e^{i \phi} \overrightarrow{p^{\prime}} \cdot \vec{\tau}$, where $\overrightarrow{p^{\prime}} \cdot \overrightarrow{p^{\prime}}=1$. To determine $\phi$ and $\overrightarrow{p^{\prime}}$, the following three relations can be used: (i) $[T, P]=0$, (ii) $P^{\dagger} P=1$ and (iii) $(T P)^{2}=-1$. Then, the general solution for $P$ is given by $P= \pm \tau_{0}$ or $P=\cos \theta \tau_{z}-\sin \theta \tau_{x}$ with $\theta \in[0,2 \pi]$.

The invariance of the Hamiltonian under $P$, that is, $H(-\mathbf{k})=P H(\mathbf{k}) P^{-1}$ combined with the TRS constrains the possible form of the Hamiltonian, which can be summarized in the following way.

(i) When $P= \pm \tau_{0} . H(\mathbf{k})=a_{0}(\mathbf{k})+\sum_{i=1}^{5} a_{i}(\mathbf{k}) \Gamma_{i}$, where $\Gamma_{1}=\tau_{x}, \Gamma_{2}=\tau_{y} \sigma_{z}$, $\Gamma_{3}=\tau_{y} \sigma_{x}, \Gamma_{4}=\tau_{y} \sigma_{y}, \Gamma_{5}=\tau_{z}$. Hence, $h_{\uparrow \uparrow}=a_{0}+a_{1} \tau_{x}+a_{2} \tau_{y}+a_{5} \tau_{z}$ and $h_{\uparrow}=$ $\left(a_{3}-i a_{4}\right) \tau_{y}$. Here $a_{0,1,2,3,4,5}(\mathbf{k})$ are all real and even under the sign change of $\mathbf{k}$.

(ii) When $P=\cos \theta \tau_{z}-\sin \theta \tau_{x} . H(\mathbf{k})=a_{0}(\mathbf{k})+\sum_{i=1}^{5} a_{i}(\mathbf{k}) \Gamma_{i}$, where $\Gamma_{1}=\mu_{x} \sigma_{z}$, $\Gamma_{2}=\mu_{y}, \Gamma_{3}=\mu_{x} \sigma_{x}, \Gamma_{4}=\mu_{x} \sigma_{y}, \Gamma_{5}=\mu_{z}$ and all $a_{0,1,2,3,45}$ are real functions.

Hence, $h_{\uparrow \uparrow}=a_{0}+a_{1} \mu_{x}+a_{2} \mu_{y}+a_{5} \mu_{z}$ and $h_{\uparrow \downarrow}=\left(a_{3}-i a_{4}\right) \mu_{x}$. Here $a_{0,5}(-\mathbf{k})=a_{0,5}(\mathbf{k}), a_{1,2,3,4}(-\mathbf{k})=-a_{1,2,3,4}(\mathbf{k})$ and $\mu_{x}=\cos \theta \tau_{x}+\sin \theta \tau_{z}, \mu_{y}=\tau_{y}$, $\mu_{z}=-\sin \theta \tau_{x}+\cos \theta \tau_{z}$.

In both cases, an $\mathrm{ABC}$ is possible only if the five equations $a_{1,2,3,4,5}=0$ are satisfied simultaneously. Here each function $a_{i}$ has four variables including the three momentum components $k_{x, y, z}$ and one external control parameter $m$, that is, $a_{i}=a_{i}\left(k_{x}, k_{y}, k_{z}, m\right)$. As the number of equations to be satisfied is five, whereas the number of variables is four, the condition for the ABC cannot be satisfied in general at a generic momentum $\mathbf{k}$. However, it is worth to note that the above consideration does not rule out the $\mathrm{ABC}$ at non-generic points in the momentum space with high symmetry. For instance, as pointed out by Murakami ${ }^{4}$, at the timereversal invariant momentum $\mathbf{k}=\mathbf{k}_{\text {TRIM }}$, where $\mathbf{k}$ and $-\mathbf{k}$ are equivalent, all odd functions in $H(\mathbf{k})$ vanish. In the case of (ii) with $P=\cos \theta \tau_{z}-\sin \theta \tau_{x}$,

$a_{1,2,3,4}\left(\mathbf{k}_{\text {TRIM }}\right)=0$. Therefore, an $\mathrm{ABC}$ is possible if and only if one condition $a_{5}\left(\mathbf{k}_{\text {TRIM }}, m\right)=0$ is satisfied, which can be achieved by tuning one external control parameter $m$. This is the reason why the topological phase transition between two insulators can occur through an $\mathrm{ABC}$ at a time-reversal invariant momentum.

Now let us show how the condition for $\mathrm{ABC}$ is modified by the presence of the additional rotation symmetry $C_{n}$ with respect to the $z$ axis. Here $n$ is restricted to be $n=2,3,4,6$ in periodic lattice systems. Using a basis in which both $\left.H\left(k_{z}\right)\right|_{k_{x}=k_{y}=0}$ and $C_{n}$ are diagonal, $C_{n}$ can be represented by a diagonal matrix

$C_{n}=\operatorname{diag}\left[u_{A, \uparrow}, u_{B, \uparrow}, u_{A, \downarrow}, u_{B, \downarrow}\right]=\operatorname{diag}\left[\alpha_{p}, \alpha_{q}, \alpha_{p}, \alpha_{s}\right]$, where $\alpha_{p}=\exp \left[i \frac{2 \pi}{n}\left(p+\frac{1}{2}\right)\right]$ with $p=0,1, \ldots, n-1$ (ref. 21). For convenience, we express $C_{n}$ in the following way:

$$
C_{n}=\left(\begin{array}{cc}
e^{i \pi\left(\frac{1+p+q}{n}+\frac{p-q}{n} \tau_{z}\right)} & 0 \\
0 & e^{i \pi\left(\frac{1+r+s}{n}+\frac{r-s_{z}}{n} \tau_{z}\right)}
\end{array}\right) .
$$

The invariance of the Hamiltonian under $C_{n}$ leads to

$$
C_{n} H\left(k_{+}, k_{-}, k_{z}\right) C_{n}^{-1}=H\left(k_{+} e^{i \frac{2 \pi}{n}}, k_{-} e^{-i \frac{2 \pi}{n}}, k_{z}\right),
$$

where $k_{ \pm}=k_{x} \pm i k_{y}$. From equation (1), we can obtain that

$$
\begin{aligned}
& e^{i \frac{\pi}{n}(p-q) \tau_{z}} h_{\uparrow \uparrow}\left(k_{ \pm}\right) e^{-i \frac{\pi}{n}(p-q) \tau_{z}}=h_{\uparrow \uparrow}\left(k_{ \pm} e^{ \pm \frac{2 \pi}{n}}\right), \\
& e^{i \frac{\pi}{n}(p+q-r-s)} e^{i \frac{\pi}{n}(p-q) \tau_{z}} h_{\uparrow \downarrow}\left(k_{ \pm}\right) e^{i \frac{\pi}{n}(s-r) \tau_{z}}=h_{\uparrow \downarrow}\left(k_{ \pm} e^{ \pm i \frac{i \pi}{n}}\right) .
\end{aligned}
$$

The equations above can be further simplified by considering the constraint on $C_{n}$ because of TRS. Namely, as $\left[T, C_{n}\right]=0$, we can show that $\exp \left[i \frac{2 \pi}{n}(p+r+1)\right]=1$, $\exp \left[i \frac{2 \pi}{n}(q+s+1)\right]=1$ and $C_{n}=\operatorname{diag}\left[\alpha_{p}, \alpha_{q}, \alpha_{p}^{*}, \alpha_{q}^{*}\right]$. Namely, $C_{n}=\operatorname{diag}\left[u_{A, \uparrow}, u_{B, \uparrow}, u_{A, \downarrow}=u_{A, \uparrow}^{*}, u_{B, \downarrow}=u_{B, \uparrow}^{*}\right]$. Then equation (2) becomes

$$
\begin{aligned}
& e^{i \frac{i \pi}{n}(p-q) \tau_{z}} h_{\uparrow \uparrow}\left(k_{ \pm}\right) e^{-i \frac{\pi}{n}(p-q) \tau_{z}}=h_{\uparrow \uparrow}\left(k_{ \pm} e^{ \pm i \frac{2 \pi}{n}}\right), \\
& e^{i \frac{i \pi}{m}(q-r)} e^{i \frac{\pi}{n}(p-q) \tau_{z}} h_{\uparrow \downarrow}\left(k_{ \pm}\right) e^{i \frac{\pi}{n}(p-q) \tau_{z}}=h_{\uparrow \downarrow}\left(k_{ \pm} e^{ \pm i \frac{2 \pi}{n}}\right) .
\end{aligned}
$$

In general, $h_{\uparrow \uparrow}(\mathbf{k})$ and $h_{\uparrow \downarrow}(\mathbf{k})$ can be represented by

$$
\begin{aligned}
& h_{\uparrow \uparrow}(\mathbf{k})=f_{0}(\mathbf{k})+f_{+}(\mathbf{k}) \tau_{+}+f_{+}^{*}(\mathbf{k}) \tau_{-}+f_{z}(\mathbf{k}) \tau_{z}, \\
& h_{\uparrow \downarrow}(\mathbf{k})=g_{0}(\mathbf{k})+g_{+}(\mathbf{k}) \tau_{+}+g_{-}(\mathbf{k}) \tau_{-}+g_{z}(\mathbf{k}) \tau_{z},
\end{aligned}
$$

where $f_{0, z}$ are real functions, whereas $f_{+}, g_{0 \pm z}$ are complex functions. Also $\tau_{ \pm}=\tau_{x} \pm i \tau_{y}$. As $f_{0}$ does not affect the gap-closing, we can neglect it in the forthcoming discussion. Equation (3) gives the following relations:

$$
\begin{aligned}
& e^{i \frac{2 \pi}{n}(p-q)} f_{+}\left(k_{ \pm}, k_{z}\right)=f_{+}\left(k_{ \pm} e^{ \pm \frac{2 \pi}{n}}, k_{z}\right), \\
& f_{z}\left(k_{ \pm}, k_{z}\right)=f_{z}\left(k_{ \pm} e^{ \pm \frac{2 \pi}{n}}, k_{z}\right),
\end{aligned}
$$

and

$$
\begin{aligned}
& e^{i \frac{2 \pi}{n}(p-r)} g_{0+z}\left(k_{ \pm}, k_{z}\right)=g_{0+z}\left(k_{ \pm} e^{ \pm \frac{2 \pi n}{n}}, k_{z}\right), \\
& e^{i \frac{2 \pi}{n}(q-s)} g_{0-z}\left(k_{ \pm}, k_{z}\right)=g_{0-z}\left(k_{ \pm} e^{ \pm \frac{2 \pi n}{n}}, k_{z}\right), \\
& e^{i \frac{2 \pi}{n}(q-r)} g_{ \pm}\left(k_{ \pm}, k_{z}\right)=g_{ \pm}\left(k_{ \pm} e^{ \pm \frac{2 \pi \pi}{n}}, k_{z}\right),
\end{aligned}
$$

where $g_{0 \pm z}=g_{0} \pm g_{z}$. The equations (5) and (6) are the key results, which lead to the full classification of the 3D Dirac SM.

For the classification, we first consider the TRS and IS, which restrict the possible structure of the Hamiltonian summarized in (i) and (ii). After that the rotational symmetry is imposed to the Hamiltonian by using the equations (5) and (6). As shown previously, the most general form of $P$ is given by $P= \pm \tau_{0}$ or $P=\cos \theta \tau_{z}-\sin \theta \tau_{x}$ with $\theta \in[0,2 \pi]$. To determine the matrix representation of $P$ and $C_{n}$, we use the basis in which both the $\left.H\left(k_{z}\right)\right|_{k_{x}=k_{y}=0}$ and $C_{n}$ are diagonal. As noted before, in such a basis, the Hamiltonian should have a diagonal form given by $\left.H\left(k_{z}\right)\right|_{k_{x}=k_{y}=0}$ with $\Gamma \in\left\{\tau_{z}, \tau_{z} \sigma_{z}\right\}$. As $d\left(k_{z}\right)$ should have a definite parity under IS as shown in (i) and (ii), the possible form of $P$ is restricted to be $P= \pm \tau_{0}, \pm \tau_{x}$, $\pm \tau_{z}$. In particular, when $P= \pm \tau_{0}$ or $\pm \tau_{z}, d(\mathbf{k})$ is even under the sign change of the momentum $\mathbf{k}$, which leads to the $3 \mathrm{D}$ topological Dirac SM via an $\mathrm{ABC}$ as summarized in Table 1 . On the other hand, when $P= \pm \tau_{x}, d(\mathbf{k})$ is odd under the sign change of $\mathbf{k}$, which gives rise to a 3D Dirac SM with a single bulk DP as summarized in Table 2.

Let us describe the constraints from the rotation symmetry in detail. When $P= \pm \tau_{0}$, the Hamiltonian $h_{\uparrow \uparrow}(\mathbf{k})$ and $h_{\uparrow \downarrow}(\mathbf{k})$ are given by

$$
\begin{aligned}
& h_{\uparrow \uparrow}(\mathbf{k})=a_{1}(\mathbf{k}) \tau_{x}+a_{2}(\mathbf{k}) \tau_{y}+a_{5}(\mathbf{k}) \tau_{z}, \\
& h_{\uparrow \downarrow}(\mathbf{k})=\left(a_{3}(\mathbf{k})-i a_{4}(\mathbf{k})\right) \tau_{y} .
\end{aligned}
$$

On the other hand, when $P= \pm \tau_{z}$,

$$
\begin{aligned}
& h_{\uparrow \uparrow}(\mathbf{k})=a_{1}(\mathbf{k}) \tau_{x}+a_{2}(\mathbf{k}) \tau_{y}+a_{5}(\mathbf{k}) \tau_{z}, \\
& h_{\uparrow \downarrow}(\mathbf{k})=\left(a_{3}(\mathbf{k})-i a_{4}(\mathbf{k})\right) \tau_{x} .
\end{aligned}
$$

In both cases, fromequations (5) and (6), we can obtain

$$
\begin{aligned}
& e^{i \frac{2 \pi}{n}(p-q)} f_{+}\left(k_{ \pm}, k_{z}\right)=f_{+}\left(k_{ \pm} e^{ \pm \frac{2 \pi}{n}}, k_{z}\right), \\
& a_{5}\left(k_{ \pm}, k_{z}\right)=a_{5}\left(k_{ \pm} e^{ \pm \frac{2 \pi}{n}}, k_{z}\right), \\
& e^{i \frac{2 \pi}{n}(q-r)} g\left(k_{ \pm}, k_{z}\right)=g\left(k_{ \pm} e^{ \pm \frac{2 \pi}{n}}, k_{z}\right),
\end{aligned}
$$

where $f_{+}(\mathbf{k})=\left(a_{1}(\mathbf{k})-i a_{2}(\mathbf{k})\right) / 2$ and $g(\mathbf{k})=\left(a_{3}(\mathbf{k})-i a_{4}(\mathbf{k})\right) / 2$. As $f_{+}$and $g$ should be zero on the $k_{z}$ axis, we obtain $e^{\frac{i 2 \pi}{n}(p-q)}=u_{A, \uparrow} u_{B \uparrow}^{*} \neq 1$ and $e^{i \frac{i \pi}{n}(q-r)}=u_{B, \uparrow} u_{A, \downarrow}^{*} \neq 1$. Hence, the ABC is possible when $\left\{u_{A, \uparrow}, u_{A, \downarrow}\right\} \cap\left\{u_{B, \uparrow}\right.$, $\left.u_{B, \downarrow}\right\}=\emptyset$, that is, when the valence and conduction bands have no rotation eigenvalue in common.

Finally, when $P= \pm \tau_{x}$, the Hamiltonian $h_{\uparrow \uparrow}(\mathbf{k})$ and $h_{\uparrow \downarrow}(\mathbf{k})$ are given by

$$
\begin{aligned}
& h_{\uparrow \uparrow}(\mathbf{k})=a_{5}(\mathbf{k}) \tau_{x}+a_{2}(\mathbf{k}) \tau_{y}+a_{1}(\mathbf{k}) \tau_{z}, \\
& h_{\uparrow \downarrow}(\mathbf{k})=\left(a_{3}(\mathbf{k})-i a_{4}(\mathbf{k})\right) \tau_{z} .
\end{aligned}
$$

From the equations (5) and (6), we can obtain

$$
\begin{aligned}
& e^{i \frac{2 \pi}{n}(p-q)} f_{+}\left(q_{ \pm}, k_{z}\right)=f_{+}\left(q_{ \pm} e^{ \pm i \frac{2 \pi}{n}}, k_{z}\right), \\
& a_{1}\left(q_{ \pm}, k_{z}\right)=a_{1}\left(q_{ \pm} e^{ \pm \frac{22 \pi}{n}}, k_{z}\right), \\
& e^{i \frac{2 \pi}{n}(p-r)} g_{z}\left(q_{ \pm}, k_{z}\right)=e^{i \frac{2 \pi}{n}(q-s)} g_{z}\left(q_{ \pm}, k_{z}\right)=g_{z}\left(q_{ \pm} e^{ \pm \frac{i 2 \pi}{n}}, k_{z}\right)
\end{aligned}
$$

where $f_{+}(\mathbf{k})=\left(a_{5}(\mathbf{k})-i a_{2}(\mathbf{k})\right) / 2$ and $g_{z}(\mathbf{k})=\left(a_{3}(\mathbf{k})-i a_{4}(\mathbf{k})\right)$. From the condition that $f_{+}=g_{z}=0$ on the $k_{z}$ axis, we obtain $e^{i \frac{2 \pi}{n}(p-q)}=u_{A, \uparrow} u_{B, \uparrow}^{*} \neq 1$, $e^{i \frac{2 \pi}{n}(p-r)}=u_{A, \uparrow} u_{A, \downarrow}^{*} \neq 1, e^{i \frac{i \pi}{n}(q-s)}=u_{B, \uparrow} u_{B, \downarrow}^{*} \neq 1$ and $u_{A, \uparrow}^{2} \stackrel{u_{B, \uparrow}}{=}$. Namely, the following two conditions should be satisfied:

$$
\left\{u_{A, \uparrow}, u_{B, \downarrow}\right\} \bigcap\left\{u_{B, \uparrow}, u_{A, \downarrow}\right\}=\emptyset
$$

$$
u_{A, \uparrow}=-u_{B, \uparrow} .
$$

The physical meaning of these two conditions is as follows. First of all, as the IS flips the orbitals in this case $\left(P= \pm \tau_{x}\right), E_{A, \sigma}\left(k_{z}\right)=E_{B, \sigma}\left(-k_{z}\right)$. Then, the combined operation of the TRS and IS ensures $E_{A \uparrow}\left(k_{z}\right)=E_{B, \downarrow}\left(k_{z}\right)$ and $E_{A, \downarrow}\left(k_{z}\right)=E_{B, \uparrow}\left(k_{z}\right)$ on the $k_{z}$ axis. Note that the orbital index is physically meaningful in this case, because the angular momentum is a good quantum number on the $k_{z}$ axis. Then, the crossing between two degenerate bands requires $\left\{u_{A, \uparrow}, u_{B, \downarrow}\right\} \cap\left\{u_{B, \uparrow}, u_{A, \downarrow}\right\}=\emptyset$. Moreover, as $\left(C_{n} P\right)^{2}=\left(P C_{n}\right)^{2}$ on the $k_{z}$ axis, $C_{n} P= \pm P C_{n}$. However, as the equation (12) is violated if $\left[C_{n}, P\right]=0$ is fulfilled, we obtain $\left\{C_{n}, P\right\}=0$, which immediately leads to the equation (13). In fact, the condition $u_{A, \uparrow}=-u_{B, \uparrow}$ means $\exp \left[i \frac{2 \pi}{n}\left(p+\frac{1}{2}\right)\right]=\exp \left[i \frac{2 \pi}{n}\left(q+\frac{1}{2}\right)\right]$ with integers $p$ and $q$. But this relation cannot be satisfied if $n=3$. Therefore, the 3D Dirac SM with a single DP cannot exist in systems with $C_{3}$ invariance. 
Absence of the symmetry-protected Dirac point at the $\Gamma$ point. Let us explain why the symmetry-protected DP cannot exist at the $\Gamma$ point in the first BZ. Here we only consider the fourfold degenerate DP, which splits into two doubly degenerate states away from the band crossing point. At the $\Gamma$ point, the representation of the space group is equivalent to the representation of the point group, which is true for both the non-symmorphic and symmorphic space groups. As the symmetry-protected DP can appear when the space group symmetry at a given momentum allows a four-dimensional irreducible representation, what we have to do is to check the presence of the four-dimensional irreducible representation in the double-valued representation of the relevant point group. It is straightforward to confirm that among the 32-point groups, only the cubic groups $T_{d}, O, O_{h}$ support a four-dimensional irreducible representation in their double-valued representation ${ }^{44}$. However, all these cubic groups contain threefold rotation axes. According to Table 2, an isolated symmetry-protected DP locating at a TRIM is forbidden if the system has a threefold rotation symmetry. Therefore, we conclude that the symmetry-protected DP cannot present at the $\Gamma$ point in $3 \mathrm{D}$ periodic electronic systems. A similar conclusion is also obtained in ref. 15 .

The emergence of the mirror symmetry in the Hamiltonian. Let us briefly describe how the mirror symmetry manifests in the effective Hamiltonian $H(\mathbf{k})=\sum_{i=1}^{5} a_{i}(\mathbf{k}) \Gamma_{i}$. As shown in Table 1 , in systems with the $C_{4}$ or $C_{6}$ symmetry, either $f(\mathbf{k})$ or $g(\mathbf{k})$ becomes zero in the $k_{z}=0$ plane. Although only the lowest order terms are shown in Table 1, we can show that the same result holds in all orders. This means that the effective Hamiltonian can be written as $H(\mathbf{k})=$ $\sum_{i=1,2,3} a_{i}^{\prime}(\mathbf{k}) \Gamma_{i}^{\prime}$ in the $k_{z}=0$ plane. Here $\Gamma_{1,2,3}^{\prime}$ are three mutually anti-commuting Gamma matrices. As only three Gamma matrices appear in the Hamiltonian, we can define a conserved quantity $\Gamma_{4}^{\prime} \Gamma_{5}^{\prime}$ satisfying $\left[H(\mathbf{k}), \Gamma_{4}^{\prime} \Gamma_{5}^{\prime}\right]=0$. It is straightforward to show that $\Gamma_{4}^{\prime} \Gamma_{5}^{\prime}$ is equivalent to the mirror operator $M$ in all cases, hence, the system has the mirror symmetry in the $k_{z}=0$ plane.

The lattice Hamiltonians. We can construct the lattice Hamiltonians straightforwardly by using the information in Tables 1 and 2. For instance, for the $C_{4}$ invariant system with $P= \pm \tau_{z}$, that is, 6th row of Table 1 , we can use

$$
\begin{aligned}
& f=\eta\left(\sin k_{x}+i \sin k_{y}\right), \\
& g=\sin k_{z}\left[(\beta+\gamma)\left(\cos k_{y}-\cos k_{x}\right)+i(\beta-\gamma) \sin k_{x} \sin k_{y}\right], \\
& a_{5}=M-t_{x y}\left(\cos k_{x}+\cos k_{y}\right)-t_{z} \cos k_{z},
\end{aligned}
$$

where $\eta, \beta, \gamma, M, t_{x y}, t_{z}$ are real constants. More explicitly,

$$
\begin{aligned}
& H=\sum_{\mathbf{k}} \eta\left[\sin k_{x} c^{\dagger}(\mathbf{k}) \tau_{x} \sigma_{z} c(\mathbf{k})-\sin k_{y} c^{\dagger}(\mathbf{k}) \tau_{y} c(\mathbf{k})\right] \\
& +\sum_{\mathbf{k}}(\beta+\gamma) \sin k_{z}\left(\cos k_{y}-\cos k_{x}\right)\left[c^{\dagger}(\mathbf{k}) \tau_{x} \sigma_{x} c(\mathbf{k})\right] \\
& +\sum_{\mathbf{k}}(-1)(\beta-\gamma) \sin k_{z} \sin k_{x} \sin k_{y}\left[c^{\dagger}(\mathbf{k}) \tau_{x} \sigma_{y} c(\mathbf{k})\right] \\
& +\sum_{\mathbf{k}}\left[M-t_{x y}\left(\cos k_{x}+\cos k_{y}\right)-t_{z} \cos k_{z}\right]\left[c^{\dagger}(\mathbf{k}) \tau_{z} c(\mathbf{k})\right],
\end{aligned}
$$

where $c^{\dagger}=\left[c_{A, \uparrow}^{\dagger}, c_{B, \uparrow}^{\dagger}, c_{A, \downarrow}^{\dagger}, c_{B, \downarrow}^{\dagger}\right]$. In real space, the Hamiltonian becomes

$$
\begin{aligned}
& H=\frac{\eta}{2} \sum_{n}\left[-i c_{n}^{\dagger} \tau_{x} \sigma_{z} c_{n+\hat{x}}+i c_{n}^{\dagger} \tau_{y} c_{n+\hat{y}}+\text { h.c. }\right] \\
& +\frac{(\beta+\gamma)}{4} \sum_{n}\left[-i c_{n}^{\dagger} \tau_{x} \sigma_{x} c_{n+\hat{y}+\hat{z}}-i c_{n}^{\dagger} \tau_{x} \sigma_{x} c_{n-\hat{y}+\hat{z}}\right. \\
& \left.+i c_{n}^{\dagger} \tau_{x} \sigma_{x} c_{n+\hat{x}+\hat{z}}+i c_{n}^{\dagger} \tau_{x} \sigma_{x} c_{n-\hat{x}+\hat{z}}+\text { h.c. }\right] \\
& +\frac{(\beta-\gamma)}{8} \sum_{n}\left[-i c_{n}^{\dagger} \tau_{x} \sigma_{y} c_{n+\hat{x}+\hat{y}+\hat{z}}-i c_{n}^{\dagger} \tau_{x} \sigma_{y} c_{n-\hat{x}-\hat{y}+\hat{z}}\right.
\end{aligned}
$$

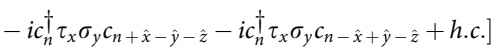

$$
\begin{aligned}
& +M \sum_{n} c_{n}^{\dagger} \tau_{z} c_{n}-\frac{t_{x y}}{2} \sum_{n}\left[c_{n}^{\dagger} \tau_{z} c_{n+\hat{x}}+c_{n}^{\dagger} \tau_{z} c_{n+\hat{y}}+\text { h.c. }\right] \\
& -\frac{t_{z}}{2} \sum_{n}\left[c_{n}^{\dagger} \tau_{z} c_{n+\hat{z}}+\text { h.c. }\right] \text {, }
\end{aligned}
$$

where $n$ indicates the lattice sites and $\hat{x}, \hat{y}, \hat{z}$ are the unit lattice vectors along $x, y, z$ directions. $\eta$ indicates the nearest neighbour hopping amplitudes in the $x y$ plane, $(\beta+\gamma)$ denotes the next nearest neighbour hopping amplitudes in the $y z$ and $z x$ planes, and $(\beta-\gamma)$ indicates the hopping process along the body-diagonal direction of the cubic lattice. $M$ indicates the on-site potential difference between the $A$ and $B$ orbitals, and $t_{x y}\left(t_{z}\right)$ describes the hopping amplitude difference in different orbitals along the $x, y$ directions (in the $z$ direction).

Similarly, for the $C_{4}$ invariant system with $P= \pm \tau_{0}$, that is, the 7th row of Table 1, we use

$$
\begin{aligned}
& f=\eta \sin k_{z}\left(\sin k_{x}+i \sin k_{y}\right), \\
& g=\left[(\beta+\gamma)\left(\cos k_{y}-\cos k_{x}\right)+i(\beta-\gamma) \sin k_{x} \sin k_{y}\right], \\
& a_{5}=M-t_{x y}\left(\cos k_{x}+\cos k_{y}\right)-t_{z} \cos k_{z}
\end{aligned}
$$

More explicitly,

$$
\begin{aligned}
H= & \sum_{k} \eta\left[\sin k_{x} \sin k_{z} c^{\dagger}(\mathbf{k}) \tau_{x} c(\mathbf{k})-\sin k_{y} \sin k_{z} c^{\dagger}(\mathbf{k}) \tau_{y} \sigma_{z} c(\mathbf{k})\right] \\
& +\sum_{\mathbf{k}}(\beta+\gamma)\left(\cos k_{y}-\cos k_{x}\right)\left[c^{\dagger}(\mathbf{k}) \tau_{y} \sigma_{x} c(\mathbf{k})\right] \\
& +\sum_{\mathbf{k}}(-1)(\beta-\gamma) \sin k_{x} \sin k_{y}\left[c^{\dagger}(\mathbf{k}) \tau_{y} \sigma_{y} c(\mathbf{k})\right] \\
& +\sum_{\mathbf{k}}\left[M-t_{x y}\left(\cos k_{x}+\cos k_{y}\right)-t_{z} \cos k_{z}\right]\left[c^{\dagger}(\mathbf{k}) \tau_{z} c(\mathbf{k})\right]
\end{aligned}
$$

In real space, the Hamiltonian becomes

$$
\begin{aligned}
H= & -\frac{\eta}{4} \sum_{n}\left[c_{n}^{\dagger} \tau_{x} c_{n+\hat{x}+\hat{z}}-c_{n}^{\dagger} \tau_{x} c_{n-\hat{x}+\hat{z}}-c_{n}^{\dagger} \tau_{y} \sigma_{z} c_{n+\hat{y}+\hat{z}}+c_{n}^{\dagger} \tau_{y} \sigma_{z} c_{n-\hat{y}+\hat{z}}+\text { h.c. }\right] \\
& -\frac{(\beta+\gamma)}{2} \sum_{n}\left[c_{n}^{\dagger} \tau_{y} \sigma_{x} c_{n+\hat{x}}-c_{n}^{\dagger} \tau_{y} \sigma_{x} c_{n+\hat{y}}+\text { h.c. }\right] \\
& +\frac{(\beta-\gamma)}{4} \sum_{n}\left[c_{n}^{\dagger} \tau_{y} \sigma_{y} c_{n+\hat{x}+\hat{y}}-c_{n}^{\dagger} \tau_{y} \sigma_{y} c_{n+\hat{x}-\hat{y}}+\text { h.c. }\right] \\
& +M \sum_{n} c_{n}^{\dagger} \tau_{z} c_{n}-\frac{t_{x y}}{2} \sum_{n}\left[c_{n}^{\dagger} \tau_{z} c_{n+\hat{x}}+c_{n}^{\dagger} \tau_{z} c_{n+\hat{y}}+\text { h.c. }\right] \\
& -\frac{t_{z}}{2} \sum_{n}\left[c_{n}^{\dagger} \tau_{z} c_{n+\hat{z}}+\text { h.c. }\right]
\end{aligned}
$$

where $(\beta+\gamma)$ indicates the nearest neighbour hopping amplitudes in the $x y$ plane, $(\beta-\gamma)(\eta)$ denotes the next nearest neighbour hopping amplitudes in the $x y$ $(y z$ and $z x)$ planes. $M, t_{x y}, t_{z}$ have the same meaning as above. In both cases, we have chosen $\eta=1, \beta=2, \gamma=1, t_{z}=1$ while varying $M$ and $t_{x y}$ for the numerical computation.

\section{References}

1. Hasan, M. Z. \& Kane, C. L. Topological insulators. Rev. Mod. Phys. 82, 3045 (2010).

2. Qi, X.-L. \& Zhang, S.-C. Topological insulators and superconductors. Rev. Mod. Phys. 83, 1057 (2011).

3. Castro Neto, A. H., Guinea, F., Peres, N. M. R., Novoselov, K. S. \& Geim, A. K The electronic properties of graphene. Rev. Mod. Phys. 81, 109 (2009).

4. Murakami, S., Iso, S., Avishai, Y., Onoda, M. \& Nagaosa, N. Tuning phase transition between quantum spin Hall and ordinary insulating phases. Phys. Rev. B 76, 205304 (2007).

5. Murakami, S. \& Kuga, S.-i. Universal phase diagrams for the quantum spin Hall systems. Phys. Rev. B 78, 165313 (2008).

6. Murakami, S. Phase transition between the quantum spin Hall and insulator phases in 3D: emergence of a topological gapless phase. New J. Phys. 9, 356 (2007).

7. Xu, S.-Y. et al. Topological phase transition and texture inversion in a tunable topological insulator. Science 332, 560-564 (2011).

8. Sato, T. et al. Unexpected mass acquisition of Dirac fermions at the quantum phase transition of a topological insulator. Nat. Phys. 7, 840 (2011).

9. Liu, Z. K. et al. Discovery of a three-dimensional topological dirac semimetal, $\mathrm{Na}_{3}$ Bi. Science 343, 864 (2014)

10. Xu, S.-Y. et al. Observation of a bulk 3D Dirac multiplet, Lifshitz transition, and nestled spin states in $\mathrm{Na}_{3} \mathrm{Bi}$. Preprint at http://arxiv.org/abs/1312.7624 (2013).

11. Neupane, M. et al. Observation of a three dimensional topological Dirac semimetal phase in high-mobility $\mathrm{Cd}_{3} \mathrm{As}_{2}$. Nat. Commun. 5, 3786 (2014).

12. Borisenko, S. et al. Experimental realization of a three-dimensional dirac semimetal. Phys. Rev. Lett. 113, 027603 (2014).

13. Jeon, S. et al. Landau quantization and quasiparticle interference in the threedimensional dirac semimetal $\mathrm{Cd}_{3} \mathrm{As}_{2}$. Preprint at http://arxiv.org/abs/1403.3446 (2014).

14. Liu, Z. K. et al. A stable three-dimensional topological Dirac semimetal $\mathrm{Cd}_{3} \mathrm{As}_{2}$ Nat. Mater. 13, 677-681 (2014).

15. Young, S. M. et al. Dirac semimetal in three dimensions. Phys. Rev. Lett. 108, 140405 (2012).

16. Steinberg, J. A. et al. Bulk Dirac points in distorted spinels. Phys. Rev. Lett. 112, 036403 (2014).

17. Wang, Z. et al. Dirac semimetal and topological phase transitions in $\mathrm{A}_{3} \mathrm{Bi}$ (A=Na, K, Rb). Phys. Rev. B 85, 195320 (2012).

18. Wang, Z., Weng, H., Wu, Q., Dai, X. \& Fang, Z. Three-dimensional Dirac semimetal and quantum transport in $\mathrm{Cd}_{3} \mathrm{As}_{2}$. Phys. Rev. B 88, 125427 (2013).

19. Mañes, J. L. Existence of bulk chiral fermions and crystal symmetry. Phys. Rev. B 85, 155118 (2012).

20. Morimoto, T. \& Furusaki, A. Weyl and Dirac semimetals with $Z_{2}$ topological charge. Phys. Rev. B 89, 235127 (2014). 
21. Fang, C., Gilbert, M. J., Dai, X. \& Bernevig, B. A. Multi-Weyl topological semimetals stabilized by point group symmetry. Phys. Rev. Lett. 108, 266802 (2012).

22. Xu, G., Weng, H., Weng, Z., Dai, X. \& Fang, Z. Chern semimetal and the quantized anomalous hall effect in $\mathrm{HgCr}_{2} \mathrm{Se}_{4}$. Phys. Rev. Lett. 107, 186806 (2011).

23. Fu, L., Kane, C. L. \& Mele, E. J. Topological insulators in three dimensions. Phys. Rev. Lett. 98, 106803 (2007).

24. Moore, J. E. \& Balents, L. Topological invariants of time-reversal-invariant band structures. Phys. Rev. B 75, 121306(R) (2007).

25. Fu, L. \& Kane, C. L. Topological insulators with inversion symmetry. Phys. Rev. B 76, 045302 (2007).

26. Fu, L. Topological crystalline insulators. Phys. Rev. Lett. 106, 106802 (2011).

27. Fang, C., Gilbert, M. J. \& Bernevig, B. A. Bulk topological invariants in noninteracting point group symmetric insulators. Phys. Rev. B 86, 115112 (2012).

28. Slager, R.-J., Mesaros, A., Juričić, V. \& Zaanen, J. The space group classification of topological band-insulators. Nat. Phys. 9, 98-102 (2013).

29. Teo, J. C. Y., Fu, L. \& Kane, C. L. Surface states and topological invariants in three-dimensional topological insulators: application to $\mathrm{Bi}_{1-x} \mathrm{Sb}_{x}$. Phys. Rev. $B$ 78, 045426 (2008)

30. Hsieh, T. H. et al. Topological crystalline insulators in the SnTe material class. Nat. Commun. 3, 982 (2012).

31. Wan, X., Turner, A. M., Vishwanath, A. \& Savrasov, S. Y. Topological semimetal and fermi-arc surface states in the electronic structure of pyrochlore iridates. Phys. Rev. B 83, 205101 (2011).

32. Balents, L. Viewpoint: Weyl electrons kiss. Physics 4, 36 (2011).

33. Witczak-Krempa, W., Chen, G., Kim, Y. B. \& Balents, L. Correlated quantum phenomena in the strong spin-orbit regime. Annu. Rev. Condens. Matter Phys. 5, 57-82 (2014).

34. Goswami, P. \& Chakravarty, S. Quantum criticality between topological and band insulators in 3+1 dimensions. Phys. Rev. Lett. 107, 196803 (2011).

35. Isobe, H. \& Nagaosa, N. Theory of a quantum critical phenomenon in a topological insulator: $(3+1)$-dimensional quantum electrodynamics in solids. Phys. Rev. B 86, 165127 (2012).

36. Isobe, H. \& Nagaosa, N. Renormalization group study of electromagnetic interaction in multi-Dirac-node systems. Phys. Rev. B 87, 205138 (2013).

37. Hosur, P., Parameswaran, S. A. \& Vishwanath, A. Charge transport in Weyl semimetals. Phys. Rev. Lett. 108, 046602 (2012)

38. Gonza'lez, F. J., Guinea, F. \& Vozmediano, M. A. H. Non-fermi liquid behavior of electrons in the half-filled honeycomb lattice (a renormalization group approach). Nucl. Phys. B 424, 595 (1994).
39. Kotov, V. N., Uchoa, B., Pereira, V. M., Guinea, F. \& Castro Neto, A. H. Electron-electron interactions in graphene: current status and perspectives. Rev. Mod. Phys. 84, 1067 (2012).

40. Son, D. T. Quantum critical point in graphene approached in the limit of infinitely strong Coulomb interaction. Phys. Rev. B 75, 235423 (2007).

41. Moon, E.-G., Xu, C., Kim, Y. B. \& Balents, L. Non-fermi-liquid and topological states with strong spin-orbit coupling. Phys. Rev. Lett. 111, 206401 (2013).

42. Yang, B.-J. et al. Theory of topological quantum phase transitions in 3D noncentrosymmetric systems. Phys. Rev. Lett. 110, 086402 (2013).

43. Yang, B.-J., Moon, E.-G., Isobe, H. \& Nagaosa, N. Quantum criticality of topological phase transitions in 3D interacting eletronic systems. Preprint at http://arxiv.org/abs/1406.2766 (2014).

44. Bradley, C. J. \& Cracknell, A. P. The Mathematical Theory of Symmetry in Solids (Clarendon Press, Oxford, 1972).

\section{Acknowledgements}

We greatly appreciate the stimulating discussion with Xi Dai. We are grateful for support from the Japan Society for the Promotion of Science (JSPS) through the 'Funding Program for World-Leading Innovative R\&D on Science and Technology (FIRST Program) and Grant-in-Aids for Scientific Research (Kiban (S), No. 24224009) from the Ministry of Education, Culture, Sports, Science and Technology (MEXT), the Strategic International Cooperative Program (Joint Research Type) from the Japan Science and Technology Agency.

\section{Author contributions}

B.-J.Y. and N.N. conceived the original ideas. B.-J.Y. did the numerics. B.-J.Y. and N.N. analysed the data and wrote the manuscript.

\section{Additional information}

Competing financial interests: The authors declare no competing financial interests.

Reprints and permission information is available online at http://npg.nature.com/ reprintsandpermissions/

How to cite this article: Yang, B.-J. and Nagosa, N. Classification of stable three-dimensional Dirac semimetals with nontrivial topology. Nat. Commun. 5:4898 doi: $10.1038 /$ ncomms5898 (2014). 